\title{
STABILITY OF GORENSTEIN CATEGORIES
}

\author{
SEAN SATHER-WAGSTAFF, TIRDAD SHARIF, AND DIANA WHITE
}

\begin{abstract}
We show that an iteration of the procedure used to define the Gorenstein projective modules over a commutative ring $R$ yields exactly the Gorenstein projective modules. Specifically, given an exact sequence of Gorenstein projective $R$-modules $G=\cdots \stackrel{\partial_{2}^{G}}{\longrightarrow} G_{1} \stackrel{\partial_{1}^{G}}{\longrightarrow} G_{0} \stackrel{\partial_{0}^{G}}{\longrightarrow} \cdots$ such that the complexes $\operatorname{Hom}_{R}(G, H)$ and $\operatorname{Hom}_{R}(H, G)$ are exact for each Gorenstein projective $R$-module $H$, the module $\operatorname{Coker}\left(\partial_{1}^{G}\right)$ is Gorenstein projective. The proof of this result hinges upon our analysis of Gorenstein subcategories of abelian categories.
\end{abstract}

\section{INTRODUCTION}

Let $R$ be a commutative ring. Building from Auslander and Bridger's work 1, 2 , on modules of finite G-dimension, Enochs and Jenda 8 and Holm 14 introduce and study the Gorenstein projective $R$-modules as the modules of the form $\operatorname{Coker}\left(\partial_{1}^{P}\right)$ for some exact sequence of projective $R$-modules

$$
P=\cdots \stackrel{\partial_{2}^{P}}{\longrightarrow} P_{1} \stackrel{\partial_{1}^{P}}{\longrightarrow} P_{0} \stackrel{\partial_{0}^{P}}{\longrightarrow} \cdots
$$

such that the complex $\operatorname{Hom}_{R}(P, Q)$ is exact for each projective $R$-module $Q$. The class of Gorenstein projective $R$-modules is denoted $\mathcal{G}(\mathcal{P}(R))$.

In this paper, we investigate the modules that arise from an iteration of this construction. To wit, let $\mathcal{G}^{2}(\mathcal{P}(R))$ denote the class of $R$-modules $M$ for which there exists an exact sequence of Gorenstein projective $R$-modules

$$
G=\cdots \stackrel{\partial_{2}^{G}}{\longrightarrow} G_{1} \stackrel{\partial_{1}^{G}}{\longrightarrow} G_{0} \stackrel{\partial_{0}^{G}}{\longrightarrow} \cdots
$$

such that the complexes $\operatorname{Hom}_{R}(G, H)$ and $\operatorname{Hom}_{R}(H, G)$ are exact for each Gorenstein projective $R$-module $H$ and $M \cong \operatorname{Coker}\left(\partial_{1}^{G}\right)$.

One checks readily that there is a containment $\mathcal{G}(\mathcal{P}(R)) \subseteq \mathcal{G}^{2}(\mathcal{P}(R))$. We answer a question from the folklore of this subject by verifying that this containment is always an equality. This is a consequence of Corollary 4.10 as is the dual version for Gorenstein injective $R$-modules; see Example 5.3

Theorem A. If $R$ is a commutative ring, then $\mathcal{G}(\mathcal{P}(R))=\mathcal{G}^{2}(\mathcal{P}(R))$.

The proof of this result is facilitated by the consideration of a more general situation. Starting with a class of $R$-modules $\mathcal{W}$, we consider the associated full subcategory $\mathcal{G}(\mathcal{W})$ of the category of $R$-modules whose objects are defined as above;

Date: July 10, 2021.

2000 Mathematics Subject Classification. 13C05, 13D02, 13D07, 18G10, 18 G15.

Key words and phrases. abelian category, Auslander classes, Bass classes, Gorenstein projectives, Gorenstein injectives, semidualizing modules, totally reflexive modules.

TS is supported by a grant from IPM,(No. 83130311). 
see Definition 4.1. Section 4 is devoted to the category-theoretic properties of $\mathcal{G}(\mathcal{W})$, those needed for the proof of Theorem $\mathrm{A}$ and others. For instance, the next result is contained in Proposition 4.11 and Theorem 4.12 .

Theorem B. Assume $\operatorname{Ext}_{R}^{i}\left(W, W^{\prime}\right)=0$ for all $W, W^{\prime} \in \mathcal{W}$ and all $i \geqslant 1$. The Gorenstein subcategory $\mathcal{G}(\mathcal{W})$ is an exact category, and it is closed under kernels of epimorphisms (or cokernels of monomorphisms) if $\mathcal{W}$ is so.

Most of the paper focuses on subcategories of an abelian category $\mathcal{A}$. The reader is encouraged to keep certain module-categories in mind. Specific examples are provided in Section 3, and we apply our results to these examples in Section 5.

\section{Categories and Resolutions}

Here we set some notation and terminology for use throughout this paper.

Definition 1.1. In this work $\mathcal{A}$ is an abelian category. We use the term "subcategory" for a "full additive subcategory that is closed under isomorphisms." Write $\mathcal{P}=\mathcal{P}(\mathcal{A})$ and $\mathcal{I}=\mathcal{I}(\mathcal{A})$ for the subcategories of projective and injective objects in $\mathcal{A}$, respectively. A subcategory $\mathcal{X}$ of $\mathcal{A}$ is exact if it is closed under direct summands and extensions.

We fix subcategories $\mathcal{X}, \mathcal{Y}, \mathcal{W}$, and $\mathcal{V}$ of $\mathcal{A}$ such that $\mathcal{W} \subseteq \mathcal{X}$ and $\mathcal{V} \subseteq \mathcal{Y}$. Write $\mathcal{X} \perp \mathcal{Y}$ if $\operatorname{Ext}_{\mathcal{A}}^{\geqslant 1}(X, Y)=0$ for each object $X$ in $\mathcal{X}$ and each object $Y$ in $\mathcal{Y}$. For an object $A$ in $\mathcal{A}$, write $A \perp \mathcal{Y}$ (resp., $\mathcal{X} \perp A$ ) if $\operatorname{Ext}_{\mathcal{A}}^{\geqslant 1}(A, Y)=0$ for each object $Y$ in $\mathcal{Y}$ (resp., if $\operatorname{Ext}_{\mathcal{A}}^{\geqslant 1}(X, A)=0$ for each object $X$ in $\mathcal{X}$ ). We say that $\mathcal{W}$ is a cogenerator for $\mathcal{X}$ if, for each object $X$ in $\mathcal{X}$, there exists an exact sequence in $\mathcal{X}$

$$
0 \rightarrow X \rightarrow W \rightarrow X^{\prime} \rightarrow 0
$$

such that $W$ is an object in $\mathcal{W}$. The subcategory $\mathcal{W}$ is an injective cogenerator for $\mathcal{X}$ if $\mathcal{W}$ is a cogenerator for $\mathcal{X}$ and $\mathcal{X} \perp \mathcal{W}$. We say that $\mathcal{V}$ is a generator for $\mathcal{Y}$ if, for each object $Y$ in $\mathcal{Y}$, there exists an exact sequence in $\mathcal{Y}$

$$
0 \rightarrow Y^{\prime} \rightarrow V \rightarrow Y \rightarrow 0
$$

such that $V$ is an object in $\mathcal{V}$. The subcategory $\mathcal{V}$ is a projective generator for $\mathcal{Y}$ if $\mathcal{V}$ is a generator for $\mathcal{Y}$ and $\mathcal{V} \perp \mathcal{Y}$.

Definition 1.2. An $\mathcal{A}$-complex is a sequence of homomorphisms in $\mathcal{A}$

$$
A=\cdots \stackrel{\partial_{n+1}^{A}}{\longrightarrow} A_{n} \stackrel{\partial_{n}^{A}}{\longrightarrow} A_{n-1} \stackrel{\partial_{n-1}^{A}}{\longrightarrow} \cdots
$$

such that $\partial_{n-1}^{A} \partial_{n}^{A}=0$ for each integer $n$; the $n$th homology object of $A$ is $\mathrm{H}_{n}(A)=$ $\operatorname{Ker}\left(\partial_{n}^{A}\right) / \operatorname{Im}\left(\partial_{n+1}^{A}\right)$. We frequently identify objects in $\mathcal{A}$ with complexes concentrated in degree 0 .

Fix an integer $i$. The $i$ th suspension of a complex $A$, denoted $\Sigma^{i} A$, is the complex with $\left(\Sigma^{i} A\right)_{n}=A_{n-i}$ and $\partial_{n}^{\Sigma^{i} A}=(-1)^{i} \partial_{n-i}^{A}$. The hard truncation $A_{\geqslant i}$ is the complex

$$
A_{\geqslant i}=\cdots \stackrel{\partial_{i+2}^{A}}{\longrightarrow} A_{i+1} \stackrel{\partial_{i+1}^{A}}{\longrightarrow} A_{i} \rightarrow 0
$$

and the hard truncations $A_{>i}, A_{\leqslant i}$, and $A_{<i}$ are defined similarly.

The complex $A$ is $\operatorname{Hom}_{\mathcal{A}}(\mathcal{X},-)$-exact if the complex $\operatorname{Hom}_{\mathcal{A}}(X, A)$ is exact for

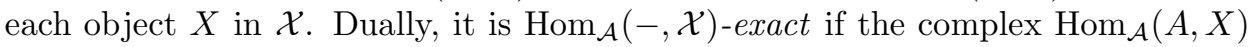
is exact for each object $X$ in $\mathcal{X}$. 
Definition 1.3. Let $A, A^{\prime}$ be $\mathcal{A}$-complexes. The Hom-complex $\operatorname{Hom}_{\mathcal{A}}\left(A, A^{\prime}\right)$ is the complex of abelian groups defined as $\operatorname{Hom}_{\mathcal{A}}\left(A, A^{\prime}\right)_{n}=\prod_{p} \operatorname{Hom}_{\mathcal{A}}\left(A_{p}, A_{p+n}^{\prime}\right)$ with $n$th differential $\partial_{n}^{\operatorname{Hom}_{\mathcal{A}}\left(A, A^{\prime}\right)}$ given by $\left\{f_{p}\right\} \mapsto\left\{\partial_{p+n}^{Y^{\prime}} f_{p}-(-1)^{n} f_{n-1} \partial_{p}^{A}\right\}$. A morphism is an element of $\operatorname{Ker}\left(\partial_{0}^{\operatorname{Hom}}{ }_{\mathcal{A}}\left(A, A^{\prime}\right)\right.$ and $\alpha$ is null-homotopic if it is in $\operatorname{Im}\left(\partial_{1}^{\operatorname{Hom}_{\mathcal{A}}\left(A, A^{\prime}\right)}\right)$. Given a second morphism $\alpha^{\prime}: A \rightarrow A^{\prime}$ we say that $\alpha$ and $\alpha^{\prime}$ are homotopic if the difference $\alpha-\alpha^{\prime}$ is null-homotopic. The morphism $\alpha$ is a homotopy equivalence if there is a morphism $\beta: A^{\prime} \rightarrow A$ such that $\beta \alpha$ is homotopic to $\operatorname{id}_{A}$ and $\alpha \beta$ is homotopic to $\operatorname{id}_{A^{\prime}}$. The complex $A$ is contractible if the identity morphism id ${ }_{A}$ is null-homotopic. When $A$ is contractible, it is exact, as is each of the complexes $\operatorname{Hom}_{\mathcal{A}}(A, N)$ and $\operatorname{Hom}_{\mathcal{A}}(M, A)$ for all objects $M$ and $N$ in $\mathcal{A}$.

A morphism of complexes $\alpha: A \rightarrow A^{\prime}$ induces homomorphisms $\mathrm{H}_{n}(\alpha): \mathrm{H}_{n}(A) \rightarrow$ $\mathrm{H}_{n}\left(A^{\prime}\right)$, and $\alpha$ is a quasiisomorphism when each $\mathrm{H}_{n}(\alpha)$ is bijective. The mapping cone of $\alpha$ is the complex $\operatorname{Cone}(\alpha)$ defined as $\operatorname{Cone}(\alpha)_{n}=A_{n}^{\prime} \oplus A_{n-1}$ with $n$th differential $\partial_{n}^{\operatorname{Cone}(\alpha)}=\left(\begin{array}{cc}\partial_{n}^{A^{\prime}} & \alpha_{n-1} \\ 0 & -\partial_{n-1}^{A}\end{array}\right)$. This definition gives a degreewise split exact sequence $0 \rightarrow A^{\prime} \rightarrow$ Cone $(\alpha) \rightarrow \Sigma A \rightarrow 0$. Further, the morphism $\alpha$ is a quasisomorphism if and only if Cone $(\alpha)$ is exact. Finally, if $\mathrm{id}_{A}$ is the identity morphism for $A$, then Cone $\left(\mathrm{id}_{A}\right)$ is contractible.

Definition 1.4. A complex $X$ is bounded if $X_{n}=0$ for $|n| \gg 0$. When $X_{-n}=0=$ $\mathrm{H}_{n}(X)$ for all $n>0$, the natural morphism $X \rightarrow \mathrm{H}_{0}(X)$ is a quasiisomorphism. In this event, $X$ is an $\mathcal{X}$-resolution of $M$ if each $X_{n}$ is an object in $\mathcal{X}$, and the following exact sequence is the augmented $\mathcal{X}$-resolution of $M$ associated to $X$.

$$
X^{+}=\cdots \stackrel{\partial_{2}^{X}}{\longrightarrow} X_{1} \stackrel{\partial_{1}^{X}}{\longrightarrow} X_{0} \rightarrow M \rightarrow 0
$$

Instead of writing "P-resolution" we will write "projective resolution." The $\mathcal{X}$ projective dimension of $M$ is the quantity

$$
\mathcal{X}-\operatorname{pd}(M)=\inf \left\{\sup \left\{n \geqslant 0 \mid X_{n} \neq 0\right\} \mid X \text { is an } \mathcal{X} \text {-resolution of } M\right\} .
$$

The objects of $\mathcal{X}$-projective dimension 0 are exactly the objects of $\mathcal{X}$. We set

$$
\text { res } \widehat{\mathcal{X}}=\text { the subcategory of objects } M \text { of } \mathcal{A} \text { with } \mathcal{X} \text { - } \operatorname{pd}(M)<\infty \text {. }
$$

We define $\mathcal{Y}$-coresolutions and $\mathcal{Y}$-injective dimension dually. The augmented $\mathcal{Y}$ coresolution associated to a $\mathcal{Y}$-coresolution $Y$ is denoted ${ }^{+} Y$, and the $\mathcal{Y}$-injective dimension of $M$ is denoted $\mathcal{Y}$ - $\operatorname{id}(M)$. We set

$$
\text { cores } \widehat{\mathcal{Y}}=\text { the subcategory of objects } N \text { of } \mathcal{A} \text { with } \mathcal{Y}-\operatorname{id}(N)<\infty \text {. }
$$

Definition 1.5. An $\mathcal{X}$-resolution $X$ is $\mathcal{X}$-proper (or simply proper) if the the augmented resolution $X^{+}$is $\operatorname{Hom}_{\mathcal{A}}(\mathcal{X},-)$-exact. We set

res $\widetilde{\mathcal{X}}=$ the subcategory of objects of $\mathcal{A}$ admitting a proper $\mathcal{X}$-resolution.

One checks readily that res $\widetilde{\mathcal{X}}$ is additive. If $X^{\prime}$ is an object in $\mathcal{X}$, then the complex $0 \rightarrow X^{\prime} \rightarrow 0$ is a proper $\mathcal{X}$-resolution of $X^{\prime}$; hence $X^{\prime}$ is in $\mathcal{X}$ and so $\mathcal{X} \subseteq$ res $\widetilde{\mathcal{X}}$.

Projective resolutions are always $\mathcal{P}$-proper, and so $\mathcal{A}$ has enough projectives if and only if res $\widetilde{\mathcal{P}}=\mathcal{A}$. If $M$ is an object in $\mathcal{A}$ that admits an $\mathcal{X}$-resolution $X \stackrel{\simeq}{\rightarrow} M$ and a projective resolution $P \stackrel{\simeq}{\rightarrow} M$, then there exists a quasiisomorphism $P \stackrel{\simeq}{\rightarrow} X$.

Proper coresolutions are defined dually, and we set

cores $\widetilde{\mathcal{Y}}=$ the subcategory of objects of $\mathcal{A}$ admitting a proper $\mathcal{Y}$-coresolution. 
Again, cores $\widetilde{\mathcal{Y}}$ is additive and $\mathcal{Y} \subseteq \operatorname{cores} \widetilde{\mathcal{Y}}$. Injective coresolutions are always $\mathcal{I}$ proper, and so $\mathcal{A}$ has enough injectives if and only if cores $\widetilde{\mathcal{I}}=\mathcal{A}$. If $N$ is an object in $\mathcal{A}$ that admits a $\mathcal{Y}$-coresolution $N \stackrel{\simeq}{\rightarrow} Y$ and an injective resolution $N \stackrel{\simeq}{\rightarrow} I$, then there exists a quasiisomorphism $Y \stackrel{\simeq}{\rightarrow} I$.

The next lemmata are standard or have standard proofs; for 1.6 see 3 , pf. of (2.3)], for 1.7 see [3, pf. of (2.1)], for [1.8 repeatedly apply Definition 1.1, and for the "Horseshoe Lemma" 1.9 see [9, pf. of (8.2.1)].

Lemma 1.6. Let $0 \rightarrow A_{1} \rightarrow A_{2} \rightarrow A_{3} \rightarrow 0$ be an exact sequence in $\mathcal{A}$.

(a) If $A_{3} \perp \mathcal{W}$, then $A_{1} \perp \mathcal{W}$ if and only if $A_{2} \perp \mathcal{W}$. If $A_{1} \perp \mathcal{W}$ and $A_{2} \perp \mathcal{W}$, then $A_{3} \perp \mathcal{W}$ if and only if the given sequence is $\operatorname{Hom}_{\mathcal{A}}(-, \mathcal{W})$ exact.

(b) If $\mathcal{V} \perp A_{1}$, then $\mathcal{V} \perp A_{2}$ if and only if $\mathcal{V} \perp A_{3}$. If $\mathcal{V} \perp A_{2}$ and $\mathcal{V} \perp A_{3}$, then $\mathcal{V} \perp A_{1}$ if and only if the given sequence is $\operatorname{Hom}_{\mathcal{A}}(\mathcal{V},-)$ exact.

Lemma 1.7. If $\mathcal{X} \perp \mathcal{Y}$, then $\mathcal{X} \perp$ res $\widehat{\mathcal{Y}}$ and cores $\widehat{\mathcal{X}} \perp \mathcal{Y}$.

Lemma 1.8. If $\mathcal{W}$ is an injective cogenerator for $\mathcal{X}$, then every object $X$ in $\mathcal{X}$ admits a proper $\mathcal{W}$-coresolution, so $\mathcal{X} \subseteq$ cores $\widetilde{\mathcal{W}}$. If $\mathcal{V}$ is a projective generator for $\mathcal{Y}$, then every object $Y$ in $\mathcal{Y}$ admits a proper $\mathcal{V}$-resolution, so $\mathcal{Y} \subseteq$ res $\widetilde{\mathcal{V}}$.

Lemma 1.9. Let $0 \rightarrow A^{\prime} \rightarrow A \rightarrow A^{\prime \prime} \rightarrow 0$ be an exact sequence in $\mathcal{A}$.

(a) Assume that $A^{\prime}$ and $A^{\prime \prime}$ admit proper $\mathcal{X}$-resolutions $X^{\prime} \stackrel{\simeq}{\rightarrow} A^{\prime}$ and $X^{\prime \prime} \cong A^{\prime \prime}$. If the given sequence is $\operatorname{Hom}_{\mathcal{A}}(\mathcal{X},-)$-exact, then $A$ is in res $\widetilde{\mathcal{X}}$ with proper $\mathcal{X}$-resolution $X \stackrel{\simeq}{\rightarrow} A$ such that there exists a commutative diagram



whose top row is degreewise split exact and $\partial_{n}^{X}=\left(\begin{array}{cc}\partial_{n}^{X^{\prime}} & f_{n} \\ 0 & \partial_{n}^{X^{\prime \prime}}\end{array}\right)$.

(b) Assume that $A^{\prime}$ and $A^{\prime \prime}$ admit proper $\mathcal{Y}$-coresolutions $A^{\prime} \stackrel{\simeq}{\rightarrow} Y^{\prime}$ and $A^{\prime \prime} \stackrel{\simeq}{\longrightarrow}$ $Y^{\prime \prime}$. If the given sequence is $\operatorname{Hom}_{\mathcal{A}}(-, \mathcal{Y})$-exact, then $A$ is in cores $\widetilde{\mathcal{Y}}$ with proper $\mathcal{Y}$-coresolution $A \stackrel{\simeq}{\rightarrow} Y$ such that there exists a commutative diagram

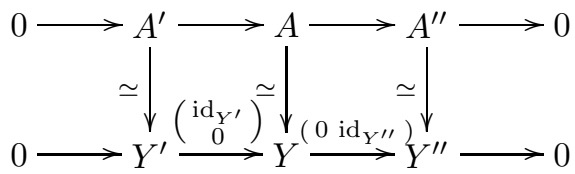

whose bottom row is degreewise split exact and $\partial_{n}^{Y}=\left(\begin{array}{cc}\partial_{n}^{Y^{\prime}} & g_{n} \\ 0 & \partial_{n}^{Y^{\prime \prime}}\end{array}\right)$.

\section{Technical Results}

This section consists of three lemmata which the reader may wish to skip during the first reading. The first result is for use in the proof of Lemma 2.2 .

Lemma 2.1. Let $0 \rightarrow N^{\prime} \rightarrow N \rightarrow N^{\prime \prime} \rightarrow 0$ be an exact sequence in $\mathcal{A}$. Assume that $\mathcal{W}$ is an injective cogenerator for $\mathcal{X}$ and $\mathcal{V}$ is a projective generator for $\mathcal{Y}$. 
(a) Assume that $N^{\prime}$ is an object in cores $\widetilde{\mathcal{X}}$, and $N^{\prime \prime}$ is an object in $\operatorname{cores} \widetilde{\mathcal{W}}$. If $N^{\prime} \perp \mathcal{W}$ and $N^{\prime \prime} \perp \mathcal{W}$, then $N$ is an object in cores $\widetilde{\mathcal{W}}$.

(b) Assume that $N^{\prime \prime}$ is an object in res $\widetilde{\mathcal{Y}}$, and $N^{\prime}$ is an object in res $\widetilde{\mathcal{V}}$. If $\mathcal{V} \perp N^{\prime}$ and $\mathcal{V} \perp N^{\prime \prime}$, then $N$ is an object in res $\widetilde{\mathcal{V}}$.

Proof. We prove part (国); the proof of part (b) is dual. Let $X^{\prime}$ be a proper $\mathcal{X}$ coresolution of $N^{\prime}$ and set $N_{i}^{\prime}=\operatorname{Ker}\left(\partial_{-i}^{X^{\prime}}\right)$ for $i \geqslant 0$, which yields an exact sequence

$$
0 \rightarrow N_{i}^{\prime} \rightarrow X_{-i}^{\prime} \rightarrow N_{i+1}^{\prime} \rightarrow 0 .
$$

Each $N_{i}^{\prime}$ is an object in cores $\widetilde{\mathcal{X}}$. By induction on $i$, Lemma 1.6(国) implies $N_{i}^{\prime} \perp \mathcal{W}$.

We will construct exact sequences

$$
\begin{array}{r}
0 \rightarrow N \rightarrow W_{0} \rightarrow N_{1} \rightarrow 0 \\
0 \rightarrow N_{1}^{\prime} \rightarrow N_{1} \rightarrow N_{1}^{\prime \prime} \rightarrow 0
\end{array}
$$

such that $\circledast_{0}$ is $\operatorname{Hom}_{\mathcal{A}}(-, \mathcal{W})$-exact, $W_{0}$ is an object in $\mathcal{W}$, and $N_{1}^{\prime \prime}$ is an object in cores $\widetilde{\mathcal{W}}$ such that $N_{1}^{\prime \prime} \perp \mathcal{W}$. Inducting on $i \geqslant 0$, this will yield exact sequences

$$
\begin{aligned}
0 & \rightarrow N_{i} \rightarrow W_{-i} \rightarrow N_{i+1} \rightarrow 0 \\
0 \rightarrow N_{i+1}^{\prime} & \rightarrow N_{i+1} \rightarrow N_{i+1}^{\prime \prime}
\end{aligned}
$$

such that $\left(\circledast_{i}\right)$ is $\operatorname{Hom}_{\mathcal{A}}(-, \mathcal{W})$-exact, $W_{i}$ is an object in $\mathcal{W}$, and $N_{i+1}^{\prime \prime}$ is an object in cores $\widetilde{\mathcal{W}}$ such that $N_{i+1}^{\prime \prime} \perp \mathcal{W}$. Splicing together the sequences $\circledast_{i}$ will then yield a proper $\mathcal{W}$-coresolution of $N_{0}=N$.

Consider the following pushout diagram whose top row is the given exact sequence and whose leftmost column is $\left(*_{0}\right)$.

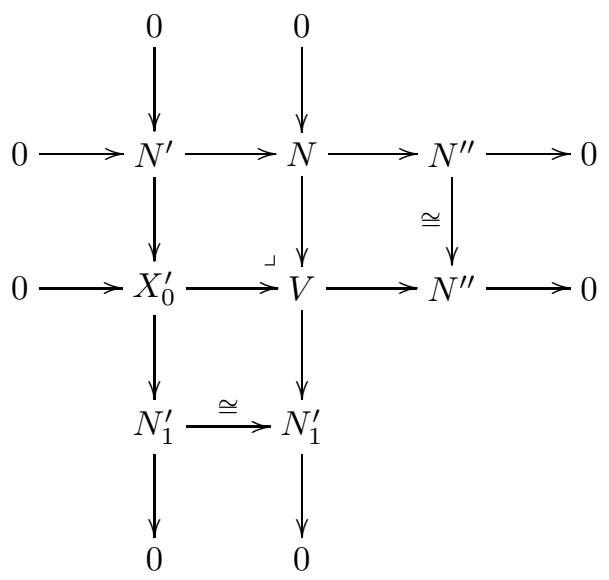

Since $N^{\prime \prime} \perp \mathcal{W}$ and $X_{0}^{\prime} \perp \mathcal{W}$, the middle row of (1) is $\operatorname{Hom}_{\mathcal{A}}(-, \mathcal{W})$-exact and $V \perp \mathcal{W}$ by Lemma 1.6(回). Note that $X_{0}^{\prime}$ is in cores $\widetilde{\mathcal{W}}$ by Lemma 1.8. Since $N^{\prime \prime}$ is in cores $\widetilde{\mathcal{W}}$, Lemma $1.9(\mathrm{~b})$ implies that $V$ is in cores $\widetilde{\mathcal{W}}$. Hence, a proper $\mathcal{W}$-coresolution of $V$ provides an exact sequence

$$
0 \rightarrow V \rightarrow W_{0} \rightarrow N_{1}^{\prime \prime} \rightarrow 0
$$

that is $\operatorname{Hom}_{\mathcal{A}}(-, \mathcal{W})$-exact with objects $W_{0}$ in $\mathcal{W}$ and $N_{1}^{\prime \prime}$ in cores $\widetilde{\mathcal{W}}$. By assumption, we have $\mathcal{X} \perp \mathcal{W}$ and $\mathcal{W} \subseteq \mathcal{X}$, and so $W_{0} \perp \mathcal{W}$ because $W_{0}$ is in $\mathcal{W}$. Since $V \perp \mathcal{W}$, Lemma1.6(国) implies $N_{1}^{\prime \prime} \perp \mathcal{W}$. With the center column of (11) this yields 
another pushout diagram, and we shall show that the middle row and the rightmost column satisfy the conditions for $\circledast_{0}$ and $t_{0}$, respectively.

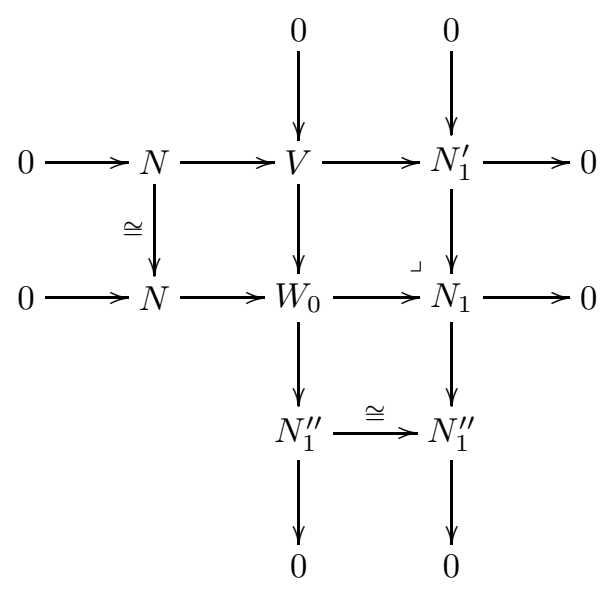

We have already seen that $W_{0}$ is in $\mathcal{W}$ and $N_{1}^{\prime \prime}$ is in cores $\widetilde{\mathcal{W}}$. Since $N_{1}^{\prime \prime} \perp \mathcal{W}$ and $N_{1}^{\prime} \perp \mathcal{W}$, the rightmost column of (2) with Lemma 1.6(可) yields $N_{1} \perp \mathcal{W}$, and so the middle row of (2) is $\operatorname{Hom}_{\mathcal{A}}(-, \mathcal{W})$-exact.

Next is a key lemma for both Theorems $\mathrm{A}$ and $\mathrm{B}$ from the introduction.

Lemma 2.2. Assume that $\mathcal{W}$ is an injective cogenerator for $\mathcal{X}$ and $\mathcal{V}$ is a projective generator for $\mathcal{Y}$.

(a) If $M$ is an object in cores $\widetilde{\mathcal{X}}$ and $M \perp \mathcal{W}$, then $M$ is in cores $\widetilde{\mathcal{W}}$.

(b) If $N$ is an object in res $\widetilde{\mathcal{Y}}$ and $\mathcal{V} \perp N$, then $N$ is in res $\widetilde{\mathcal{V}}$.

Proof. We prove part (国); the proof of part (b) is dual. Let $M \stackrel{\simeq}{\rightarrow} X$ be a proper $\mathcal{X}$-coresolution of $M$. Setting $M^{\prime}=\operatorname{Im}\left(\partial_{0}^{X}\right)$ yields an exact sequence

$$
0 \rightarrow M \rightarrow X_{0} \rightarrow M^{\prime} \rightarrow 0
$$

that is $\operatorname{Hom}_{\mathcal{A}}(-, \mathcal{X})$-exact. Since $\mathcal{W}$ is a cogenerator for $\mathcal{X}$ there is an exact sequence with objects $W_{0}$ in $\mathcal{W}$ and $X^{\prime}$ in $\mathcal{X}$.

$$
0 \rightarrow X_{0} \rightarrow W_{0} \rightarrow X^{\prime} \rightarrow 0
$$

Consider the pushout diagram whose top row is (3) and whose middle column is (4).

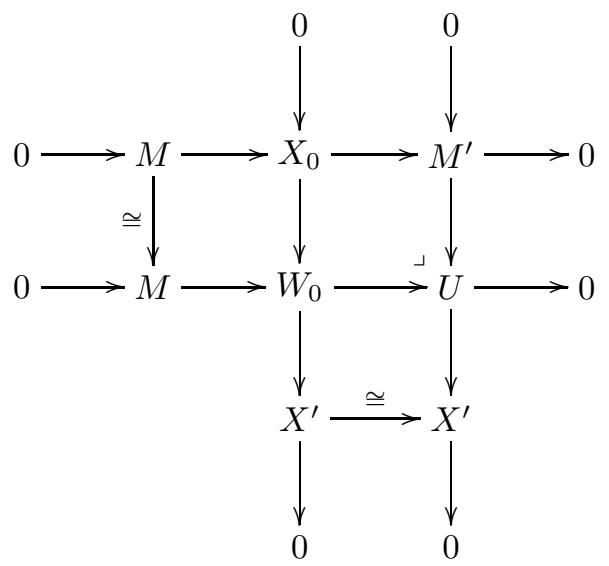


We will show that $U$ is an object in cores $\widetilde{\mathcal{W}}$ and that the middle row of (5) is $\operatorname{Hom}_{\mathcal{A}}(-, \mathcal{W})$-exact. It will then follow that a proper $\mathcal{W}$-coresolution for $M$ can be obtained by splicing the middle row of (5) with a proper $\mathcal{W}$-coresolution of $U$.

The object $M^{\prime}$ is in cores $\widetilde{\mathcal{X}}$ by construction, and $X^{\prime}$ and $X_{0}$ are in $\mathcal{X}$. Thus, $X^{\prime}$ is an object in cores $\widetilde{\mathcal{W}}$ by Lemma 1.8, and $X^{\prime} \perp \mathcal{W}$ and $X_{0} \perp \mathcal{W}$ by hypothesis. Since the top row of (5) is $\operatorname{Hom}_{\mathcal{A}}(-, \mathcal{X})$-exact, the assumption $\mathcal{W} \subseteq \mathcal{X}$ implies that the top row of (5) is $\operatorname{Hom}_{\mathcal{A}}(-, \mathcal{W})$-exact. Hence, the assumption $M \perp \mathcal{W}$ yields $M^{\prime} \perp \mathcal{W}$ by Lemma 1.6(国). With the rightmost column of (5), Lemma 2.1(国) implies that $U$ is an object in cores $\widetilde{\mathcal{W}}$. Since $X^{\prime} \perp \mathcal{W}$ and $M^{\prime} \perp \mathcal{W}$, Lemma 1.6(a) yields $U \perp \mathcal{W}$, and so the middle row of (5) is $\operatorname{Hom}_{\mathcal{A}}(-, \mathcal{W})$-exact.

The last result in this section is a tool for Proposition 4.6 .

Lemma 2.3. For $n=0,1,2, \ldots, t$, let $\mathcal{X}_{n}$ and $\mathcal{Y}_{n}$ be subcategories of $\mathcal{A}$.

(a) Assume that $\mathcal{X}_{n}$ is a cogenerator for $\mathcal{X}_{n+1}$ for each $n \geqslant 0$ and $\mathcal{X}_{t} \perp \mathcal{X}_{0}$. If $\mathcal{X}_{t}$ is closed under extensions, then $\mathcal{X}_{0}$ is an injective cogenerator for $\mathcal{X}_{t}$.

(b) Assume that $\mathcal{Y}_{n}$ is a generator for $\mathcal{Y}_{n+1}$ for each $n \geqslant 0$ and $\mathcal{Y}_{0} \perp \mathcal{Y}_{t}$. If $\mathcal{Y}_{t}$ is closed under extensions, then $\mathcal{Y}_{0}$ is a projective generator for $\mathcal{Y}_{t}$.

Proof. We prove part (国); the proof of part (b) is dual. Since $\mathcal{X}_{t} \perp \mathcal{X}_{0}$ by assumption, it remains to show that $\mathcal{X}_{0}$ is a cogenerator for $\mathcal{X}_{t}$. Fix an object $X_{t}$ in $\mathcal{X}_{t}$. By reverse induction on $i<t$, we will construct exact sequences

$$
0 \rightarrow X_{t} \rightarrow X_{i} \rightarrow X_{t}^{(i)} \rightarrow 0
$$

with objects $X_{i}$ in $\mathcal{X}_{i}$ and $X_{t}^{(i)}$ in $\mathcal{X}_{t}$. Since $\mathcal{X}_{t-1}$ is a cogenerator for $\mathcal{X}_{t}$, the sequence $\left(*_{t-1}\right)$ is known to exist. By induction, we assume that $\left(*_{i}\right)$ has been constructed and construct $\left(*_{i-1}\right)$ from it. From $\left(*_{i}\right)$ we have the object $X_{i}$ in $\mathcal{X}_{i}$. Since $\mathcal{X}_{i-1}$ is a cogenerator for $\mathcal{X}_{i}$, there is an exact sequence

$$
0 \rightarrow X_{i} \rightarrow X_{i-1} \rightarrow X_{i}^{\prime} \rightarrow 0
$$

with $X_{i-1}$ in $\mathcal{X}_{i-1}$ and $X_{i}^{\prime}$ in $\mathcal{X}_{i}$. Consider the pushout diagram

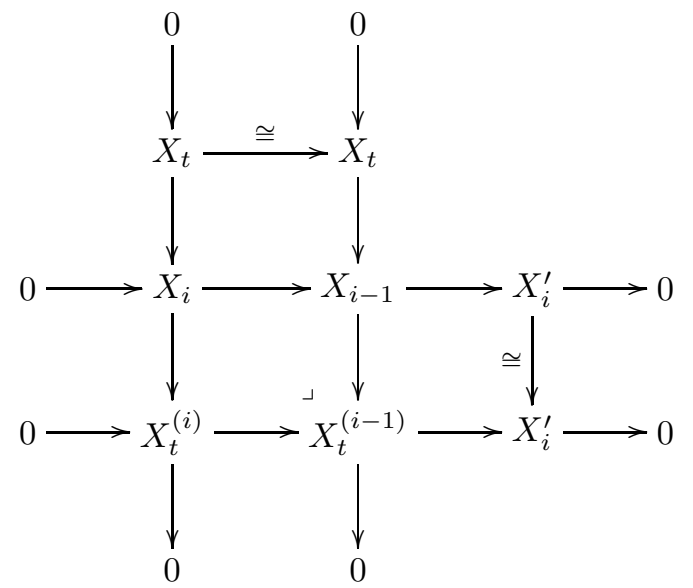

whose leftmost column is $\left(*_{i}\right)$ and whose middle row is $\left(\circledast_{i}\right)$. The object $X_{i}^{\prime}$ is in $\mathcal{X}_{i}$, and hence in $\mathcal{X}_{t}$. Since $X_{t}^{(i)}$ is also in $\mathcal{X}_{t}$, the exactness of the bottom row, with the fact that $\mathcal{X}_{t}$ is closed under extensions, implies that $X_{t}^{(i-1)}$ is in $\mathcal{X}_{t}$, so the center column of the diagram is the desired sequence $\left(*_{i-1}\right)$. 


\section{CAtegories of Interest}

Much of the motivation for this work comes from module categories. In reading this paper, the reader may find it helpful to keep in mind the examples outlined in the next few paragraphs, wherein $R$ is a commutative ring.

Definition 3.1. Let $\mathcal{M}(R)$ denote the category of $R$-modules. To be clear, we write $\mathcal{P}(R)$ for the subcategory of projective $R$-modules and $\mathcal{I}(R)$ for the subcategory of injective $R$-modules. If $\mathcal{X}(R)$ is a subcategory of $\mathcal{M}(R)$, then $\mathcal{X}^{f}(R)$ is the subcategory of finitely generated modules in $\mathcal{X}(R)$. Also set $\mathcal{A} b=\mathcal{M}(\mathbb{Z})$, the category of abelian groups.

The study of semidualizing modules was initiated independently (with different names) by Foxby [10, Golod [13], and Vasconcelos [19].

Definition 3.2. An $R$-module $C$ is semidualizing if it satisfies the following.

(1) $C$ admits a (possibly unbounded) resolution by finite rank free $R$-modules.

(2) The natural homothety map $R \rightarrow \operatorname{Hom}_{R}(C, C)$ is an isomorphism.

(3) $\operatorname{Ext}_{R}^{\geqslant 1}(C, C)=0$.

A finitely generated projective $R$-module of rank 1 is semidualizing. If $R$ is CohenMacaulay, then $C$ is dualizing if it is semidualizing and $\operatorname{id}_{R}(C)$ is finite.

Based on the work of Enochs and Jenda 8], the following notions were introduced and studied in this generality by Holm and Jørgensen [15] and White 20].

Definition 3.3. Let $C$ be a semidualizing $R$-module, and set

$$
\begin{aligned}
& \mathcal{P}_{C}(R)=\text { the subcategory of modules } P \otimes_{R} C \text { where } P \text { is } R \text {-projective } \\
& \mathcal{I}_{C}(R)=\text { the subcategory of modules } \operatorname{Hom}_{R}(C, I) \text { where } I \text { is } R \text {-injective. }
\end{aligned}
$$

Modules in $\mathcal{P}_{C}(R)$ and $\mathcal{I}_{C}(R)$ are called $C$-projective and $C$-injective, respectively. A complete $\mathcal{P} \mathcal{P}_{C}$-resolution is a complex $X$ of $R$-modules satisfying the following.

(1) $X$ is exact and $\operatorname{Hom}_{R}\left(-, \mathcal{P}_{C}(R)\right)$-exact.

(2) $X_{i}$ is projective if $i \geqslant 0$ and $X_{i}$ is $C$-projective if $i<0$.

An $R$-module $M$ is $G_{C}$-projective if there exists a complete $\mathcal{P} \mathcal{P}_{C}$-resolution $X$ such that $M \cong \operatorname{Coker}\left(\partial_{1}^{X}\right)$, in which case $X$ is a complete $\mathcal{P} \mathcal{P}_{C}$-resolution of $M$. We set

$$
\mathcal{G} \mathcal{P}_{C}(R)=\text { the subcategory of } \mathrm{G}_{C} \text {-projective } R \text {-modules. }
$$

Projective $R$-modules and $C$-projective $R$-modules are $\mathrm{G}_{C}$-projective, and $\mathcal{P}_{C}(R)$ is an injective cogenerator for $\mathcal{G P}_{C}(R)$ by [15, (2.5),(2.13)] and [20, (3.2),(3.9)].

A complete $\mathcal{I}_{C} \mathcal{I}$-coresolution is a complex $Y$ of $R$-modules such that:

(1) $Y$ is exact and $\operatorname{Hom}_{R}\left(\mathcal{I}_{C}(R),-\right)$-exact.

(2) $Y_{i}$ is injective if $i \leqslant 0$ and $Y_{i}$ is $C$-injective if $i>0$.

An $R$-module $N$ is $G_{C}$-injective if there exists a complete $\mathcal{I}_{C} \mathcal{I}$-coresolution $Y$ such that $N \cong \operatorname{Ker}\left(\partial_{0}^{Y}\right)$, in which case $Y$ is a complete $\mathcal{I}_{C} \mathcal{I}$-coresolution of $N$. We set

$$
\mathcal{G I}_{C}(R)=\text { the subcategory of } \mathrm{G}_{C} \text {-injective } R \text {-modules. }
$$

An $R$-module that is injective or $C$-injective is $\mathrm{G}_{C}$-injective, and $\mathcal{I}_{C}(R)$ is a projective generator for $\mathcal{G I}_{C}(R)$ by [15, (2.6),(2.13)] and results dual to [20, (3.2),(3.9)].

The next definition was first introduced by Auslander and Bridger [1, 2] in the case $C=R$, and in this generality by Golod [13] and Vasconcelos [19]. 
Definition 3.4. Assume that $R$ is noetherian and $C$ is a semidualizing $R$-module. A finitely generated $R$-module $H$ is totally $C$-reflexive if

(1) $\operatorname{Ext}_{R}^{\geqslant 1}(H, C)=0=\operatorname{Ext}_{R}^{\geqslant 1}\left(\operatorname{Hom}_{R}(H, C), C\right)$, and

(2) the natural biduality map $H \rightarrow \operatorname{Hom}_{R}\left(\operatorname{Hom}_{R}(H, C), C\right)$ is an isomorphism.

Each finitely generated $R$-module that is either projective or $C$-projective is totally $C$-reflexive. We set

$$
\mathcal{G}_{C}(R)=\text { the subcategory of totally } C \text {-reflexive } R \text {-modules }
$$

and $\mathcal{G}(R)=\mathcal{G}_{R}(R)$. The equality $\mathcal{G}_{C}(R)=\mathcal{G P}_{C}^{f}(R)$ is by [20, $\left.(5.4)\right]$, and $\mathcal{P}_{C}^{f}(R)$ is an injective cogenerator for $\mathcal{G}_{C}(R)$ by [20, (3.9),(5.3),(5.4)].

Over a noetherian ring, the next categories were introduced by Avramov and Foxby 4 when $C$ is dualizing, and by Christensen 7 for arbitrary $C$. (Note that these works (and others) use the notation $\mathcal{A}_{C}(R)$ and $\mathcal{B}_{C}(R)$ for certain categories of complexes, while our categories consist precisely of the modules in these other categories.) In the non-noetherian setting, these definitions are from [16, 20.

Definition 3.5. Let $C$ be a semidualizing $R$-module. The Auslander class of $C$ is the subcategory $\mathcal{A}_{C}(R)$ of $R$-modules $M$ such that

(1) $\operatorname{Tor}_{\geqslant 1}^{R}(C, M)=0=\operatorname{Ext}_{R}^{\geqslant 1}\left(C, C \otimes_{R} M\right)$, and

(2) The natural map $M \rightarrow \operatorname{Hom}_{R}\left(C, C \otimes_{R} M\right)$ is an isomorphism.

The Bass class of $C$ is the subcategory $\mathcal{B}_{C}(R)$ of $R$-modules $N$ such that

(1) $\operatorname{Ext}_{R}^{\geqslant 1}(C, M)=0=\operatorname{Tor}_{\geqslant 1}^{R}\left(C, \operatorname{Hom}_{R}(C, M)\right)$, and

(2) The natural evaluation map $C \otimes_{R} \operatorname{Hom}_{R}(C, N) \rightarrow N$ is an isomorphism.

For a discussion of the next subcategory, consult [9, Sec. 5.3].

Definition 3.6. The category of flat cotorsion $R$-modules is the subcategory $\mathcal{F}^{\prime}(R)$ of flat $R$-modules $F$ such that $\operatorname{Ext}_{R}^{\geqslant 1}\left(F^{\prime}, F\right)=0$ for each flat $R$-module $F^{\prime}$.

Gerko [12] introduced our final subcategory of interest.

Definition 3.7. Assume that $(R, \mathfrak{m}, k)$ is local and noetherian. The complexity of a finitely generated $R$-module $M$ is

$\operatorname{cx}_{R}(M)=\inf \left\{d \in \mathbb{N} \mid\right.$ there exists $c>0$ such that $\beta_{n}^{R}(M) \leqslant c n^{d-1}$ for $\left.n \gg 0\right\}$ where $\beta_{n}^{R}(M)=\operatorname{rank}_{k}\left(\operatorname{Tor}_{i}^{R}(M, k)\right)$ is the $n$th Betti number of $M$. Let $\mathcal{G}^{\prime}(R)$ denote the subcategory of modules in $\mathcal{G}(R)$ with finite complexity.

\section{Gorenstein Subchtegories}

In this section, we introduce and study the Gorenstein subcategory $\mathcal{G}(\mathcal{X})$.

Definition 4.1. An exact complex in $\mathcal{X}$ is totally $\mathcal{X}$-acyclic if it is $\operatorname{Hom}_{\mathcal{A}}(\mathcal{X},-)$ exact and $\operatorname{Hom}_{\mathcal{A}}(-, \mathcal{X})$-exact. Let $\mathcal{G}(\mathcal{X})$ denote the subcategory of $\mathcal{A}$ whose objects are of the form $M \cong \operatorname{Coker}\left(\partial_{1}^{X}\right)$ for some totally $\mathcal{X}$-acyclic complex $X$; we say that $X$ is a complete $\mathcal{X}$-resolution of $M$. Note that the isomorphisms

$$
\begin{aligned}
\operatorname{Hom}_{\mathcal{A}}\left(X^{\prime} \oplus X^{\prime \prime}, X\right) \cong \operatorname{Hom}_{\mathcal{A}}\left(X^{\prime}, X\right) \oplus \operatorname{Hom}_{\mathcal{A}}\left(X^{\prime \prime}, X\right) \\
\operatorname{Hom}_{\mathcal{A}}\left(X, X^{\prime} \oplus X^{\prime \prime}\right) \cong \operatorname{Hom}_{\mathcal{A}}\left(X, X^{\prime}\right) \oplus \operatorname{Hom}_{\mathcal{A}}\left(X, X^{\prime \prime}\right)
\end{aligned}
$$

show that the direct sum of totally $\mathcal{X}$-acyclic $\mathcal{X}$-complexes is totally $\mathcal{X}$-acyclic, and hence $\mathcal{G}(\mathcal{X})$ is additive. Set $\mathcal{G}^{0}(\mathcal{X})=\mathcal{X}$ and $\mathcal{G}^{1}(\mathcal{X})=\mathcal{G}(\mathcal{X})$, and inductively set $\mathcal{G}^{n+1}(\mathcal{X})=\mathcal{G}\left(\mathcal{G}^{n}(\mathcal{X})\right)$ for $n \geqslant 1$. 
Remark 4.2. Any contractible $\mathcal{X}$-complex is totally $\mathcal{X}$-acyclic; see 1.3 . In particular, for any object $X$ in $\mathcal{X}$, the complex

$$
0 \rightarrow X \stackrel{\operatorname{id}_{X}}{\longrightarrow} X \rightarrow 0
$$

is a complete $\mathcal{X}$-resolution, and so $X$ is an object in $\mathcal{G}(\mathcal{X})$. Hence, $\mathcal{X} \subseteq \mathcal{G}(\mathcal{X})$, and inductively $\mathcal{G}^{n}(\mathcal{X}) \subseteq \mathcal{G}^{n+1}(\mathcal{X})$ for each $n \geqslant 0$.

There is a containment $\mathcal{G}(\mathcal{X}) \subseteq$ res $\widetilde{\mathcal{X}} \cap \operatorname{cores} \widetilde{\mathcal{X}}$. Indeed, If $M$ is an object in $\mathcal{G}(\mathcal{X})$ with complete $\mathcal{X}$-resolution $X$, then the hard truncation $X_{\geqslant 0}$ is a proper $\mathcal{X}$-resolution of $M$ and $X_{<0}$ is a proper $\mathcal{X}$-coresolution of $M$.

The orthogonality properties documented next will be very useful in the sequel; compare to [6, (4.2.5)].

Proposition 4.3. If $\mathcal{X} \perp \mathcal{W}$ and $\mathcal{V} \perp \mathcal{Y}$, then $\mathcal{G}^{n}(\mathcal{X}) \perp$ res $\widehat{\mathcal{W}}$ and cores $\widehat{\mathcal{V}} \perp \mathcal{G}^{n}(\mathcal{Y})$ for each $n \geqslant 1$. In particular, if $\mathcal{W} \perp \mathcal{W}$, then $\mathcal{G}^{n}(\mathcal{W}) \perp \operatorname{res} \widehat{\mathcal{W}}$ and cores $\widehat{\mathcal{W}} \perp$ $\mathcal{G}^{n}(\mathcal{W})$ for each $n \geqslant 1$.

Proof. Assuming $\mathcal{X} \perp \mathcal{W}$, we will show $\mathcal{G}(\mathcal{X}) \perp \mathcal{W}$; the conclusion $\mathcal{G}^{n}(\mathcal{X}) \perp \mathcal{W}$ will follow by induction, and $\mathcal{G}^{n}(\mathcal{X}) \perp$ res $\widehat{\mathcal{W}}$ will then follow from Lemma 1.7. The other conclusion is verified dually. Let $M$ be an object in $\mathcal{G}(\mathcal{X})$ with complete $\mathcal{X}$ resolution $X$, and let $W$ be an object in $\mathcal{W}$. For each integer $i$ set $M_{i}=\operatorname{Coker}\left(\partial_{i}^{X}\right)$. Note that $M \cong M_{1}$. The exact sequence

$$
0 \rightarrow M_{i+1} \stackrel{\epsilon_{i}}{\longrightarrow} X_{i-1} \rightarrow M_{i} \rightarrow 0
$$

is $\operatorname{Hom}_{\mathcal{A}}(-, \mathcal{X})$-exact, and so it is $\operatorname{Hom}_{\mathcal{A}}(-, W)$-exact. In particular, the map $\operatorname{Hom}_{\mathcal{A}}\left(\epsilon_{i}, W\right)$ is surjective. Since $\mathcal{X} \perp \mathcal{W}$, part of the beginning of the associated long exact sequence in $\operatorname{Ext}_{\mathcal{A}}(-, W)$ is

$$
\operatorname{Hom}_{\mathcal{A}}\left(X_{i-1}, W\right) \stackrel{\operatorname{Hom}_{\mathcal{A}}\left(\epsilon_{i}, W\right)}{\longrightarrow} \operatorname{Hom}_{\mathcal{A}}\left(M_{i+1}, W\right) \rightarrow \operatorname{Ext}_{\mathcal{A}}^{1}\left(M_{i}, W\right) \rightarrow 0
$$

so the surjectivity of $\operatorname{Hom}_{\mathcal{A}}\left(\epsilon_{i}, W\right)$ implies $\operatorname{Ext}_{\mathcal{A}}^{1}\left(M_{i}, W\right)=0$. In particular,

$$
\operatorname{Ext}_{\mathcal{A}}^{1}(M, W) \cong \operatorname{Ext}_{\mathcal{A}}^{1}\left(M_{1}, W\right)=0 .
$$

If $j \geqslant 2$, then the remainder of the long exact sequence yields isomorphisms $\operatorname{Ext}_{\mathcal{A}}^{j}\left(M_{i}, W\right) \cong \operatorname{Ext}_{\mathcal{A}}^{j-1}\left(M_{i+1}, W\right)$. Inductively, this yields the second isomorphism in the next sequence and the desired vanishing

$$
\operatorname{Ext}_{\mathcal{A}}^{j}(M, W) \cong \operatorname{Ext}_{\mathcal{A}}^{j}\left(M_{1}, W\right) \cong \operatorname{Ext}_{\mathcal{A}}^{1}\left(M_{j}, W\right)=0 .
$$

We next present a "Horseshoe Lemma" for complete $\mathcal{X}$-resolutions; compare to [6, (4.3.5.a)].

Proposition 4.4. Consider an exact sequence in $\mathcal{A}$

$$
0 \rightarrow M^{\prime} \rightarrow M \rightarrow M^{\prime \prime} \rightarrow 0
$$

that is $\operatorname{Hom}_{\mathcal{A}}(\mathcal{X},-)$-exact and $\operatorname{Hom}_{\mathcal{A}}(-, \mathcal{X})$-exact. If $M^{\prime}$ and $M^{\prime \prime}$ are objects in $\mathcal{G}(X)$, then so is $M$. Furthermore, given complete $\mathcal{X}$-resolutions $X^{\prime}$ and $X^{\prime \prime}$ of $M^{\prime}$ and $M^{\prime \prime}$, respectively, there is a degreewise split exact sequence of complexes

$$
0 \rightarrow X^{\prime} \rightarrow X \rightarrow X^{\prime \prime} \rightarrow 0
$$

such that $X$ is a complete $\mathcal{X}$-resolution of $M$, the induced sequence

$$
0 \rightarrow \operatorname{Coker}\left(\partial_{1}^{X^{\prime}}\right) \rightarrow \operatorname{Coker}\left(\partial_{1}^{X}\right) \rightarrow \operatorname{Coker}\left(\partial_{1}^{X^{\prime \prime}}\right) \rightarrow 0
$$


is equivalent to the original sequence, and $\partial_{n}^{X}=\left(\begin{array}{cc}\partial_{n}^{X^{\prime}} & f_{n} \\ 0 & \partial_{n}^{X^{\prime \prime}}\end{array}\right)$ for each $n \in \mathbb{Z}$.

Proof. Let $X^{\prime}$ and $X^{\prime \prime}$ be complete $\mathcal{X}$-resolutions for $M^{\prime}$ and $M^{\prime \prime}$, respectively. Lemma 1.9 yields a degreewise split exact sequence of complexes $0 \rightarrow X^{\prime} \rightarrow X \rightarrow$ $X^{\prime \prime} \rightarrow 0$ such that $M \cong \operatorname{Coker}\left(\partial_{1}^{X}\right)$. Note that each $X_{i} \cong X_{i}^{\prime} \oplus X_{i}^{\prime \prime}$ is in $\mathcal{X}$. Since the complexes $X^{\prime}$ and $X^{\prime \prime}$ are both $\operatorname{Hom}_{\mathcal{A}}(\mathcal{X},-)$-exact and $\operatorname{Hom}_{\mathcal{A}}(\mathcal{X},-)$-exact, the same is true of $X$. So, $X$ is a complete $\mathcal{X}$-resolution of $M$.

Corollary 4.5. If $\mathcal{W} \perp \mathcal{W}$, then $\mathcal{G}(\mathcal{W})$ is closed under extensions.

Proof. Proposition 4.3 implies $\mathcal{G}(\mathcal{W}) \perp \mathcal{W}$ and $\mathcal{W} \perp \mathcal{G}(\mathcal{W})$. Hence, any exact sequence $0 \rightarrow M^{\prime} \rightarrow M \rightarrow M^{\prime \prime} \rightarrow 0$ with $M^{\prime}$ and $M^{\prime \prime}$ objects in $\mathcal{G}(\mathcal{W})$ is $\operatorname{Hom}_{\mathcal{A}}(\mathcal{W},-)$-exact and $\operatorname{Hom}_{\mathcal{A}}(-, \mathcal{W})$-exact. Now apply Proposition 4.4

It is unclear in general whether $\mathcal{W}$ is an injective cogenerator for $\mathcal{G}^{n}(\mathcal{X})$ without the extra hypotheses in our next result.

Proposition 4.6. Fix an integer $n \geqslant 1$.

(a) If $\mathcal{W}$ is an injective cogenerator for $\mathcal{X}$ and $\mathcal{G}^{n}(\mathcal{X})$ is closed under extensions, then $\mathcal{W}$ is an injective cogenerator for $\mathcal{G}^{n}(\mathcal{X})$.

(b) If $\mathcal{V}$ is a projective generator for $\mathcal{Y}$ and $\mathcal{G}^{n}(\mathcal{Y})$ is closed under extensions, then $\mathcal{V}$ is a projective generator for $\mathcal{G}^{n}(\mathcal{Y})$.

Proof. We prove part (a) ; the proof of part (ab) is dual. Set $\mathcal{X}_{0}=\mathcal{W}, \mathcal{X}_{1}=\mathcal{X}$, and $\mathcal{X}_{t}=\mathcal{G}^{t-1}(\mathcal{X})$ for $t=2, \ldots, n+1$. By Proposition 4.3 we know $\mathcal{G}^{n}(\mathcal{X}) \perp \mathcal{W}$, so the desired conclusion follows from Lemma 2.3

In Section 5 we document the consequences of the following result for the examples of Section 3 .

Corollary 4.7. If $\mathcal{W} \perp \mathcal{W}$, then $\mathcal{W}$ is both an injective cogenerator and a projective generator for $\mathcal{G}(\mathcal{W})$.

Proof. This follows from Corollary 4.5 and Proposition 4.6.

The next result extends part of Remark 4.2 and represents a first step in the proof of Theorem $\mathrm{A}$ from the introduction.

Theorem 4.8. Assume that $\mathcal{W}$ is an injective cogenerator for $\mathcal{X}$ and that $\mathcal{V}$ is a projective generator for $\mathcal{Y}$.

(a) If $\mathcal{X}$ is closed under extensions, then $\mathcal{G}^{n}(\mathcal{X}) \subseteq$ cores $\widetilde{\mathcal{W}}$ for each $n \geqslant 0$.

(b) If $\mathcal{Y}$ is closed under extensions, then $\mathcal{G}^{n}(\mathcal{Y}) \subseteq$ res $\widetilde{\mathcal{V}}$ for each $n \geqslant 0$.

Proof. We prove part (a) by induction on $n$; the proof of part (b) is dual. The case $n=0$ is in Lemma 1.8. When $n=1$, note that an object $M$ in $\mathcal{G}(\mathcal{X})$ is in cores $\widetilde{\mathcal{X}}$ by Remark 4.2 and one has $M \perp \mathcal{W}$ by Proposition 4.3. now apply Lemma 2.2.

Assume $n>1$ and $\mathcal{G}^{n-1}(\mathcal{X}) \subseteq$ cores $\widetilde{\mathcal{W}}$. Fix an object $M$ in $\mathcal{G}^{n}(\mathcal{X})$ and a complete $\mathcal{G}^{n-1}(\mathcal{X})$-resolution $G$ of $M$. By definition, the complex $G$ is exact and there is an isomorphism $M \cong \operatorname{Ker}\left(\partial_{-1}^{G}\right)$. For each integer $j$, set $M_{j}=\operatorname{Ker}\left(\partial_{j}^{G}\right)$ and observe that each $M_{j}$ is an object in $\mathcal{G}^{n}(\mathcal{X})$. Since each object $G_{j}$ is in $\mathcal{G}^{n-1}(\mathcal{X})$, Proposition 4.3 implies $M_{j} \perp \mathcal{W}$ and $G_{j} \perp \mathcal{W}$ for each integer $j$, and we consider the exact sequences

$$
0 \rightarrow M_{j} \rightarrow G_{j} \rightarrow M_{j-1} \rightarrow 0
$$


Our induction assumption implies that each object $G_{j}$ is in cores $\widetilde{\mathcal{W}}$.

By induction on $i \geqslant 0$, we construct exact sequences in $\mathcal{A}$

$$
\begin{aligned}
0 \rightarrow M & \rightarrow W_{0} \rightarrow W_{-1} \rightarrow \cdots \rightarrow W_{-i} \rightarrow U_{-i} \rightarrow 0 \\
& 0 \rightarrow M_{-(i+2)} \rightarrow U_{-i} \rightarrow V_{-i} \rightarrow 0 \\
& \rightarrow U_{-i} \rightarrow W_{-(i+1)} \rightarrow U_{-(i+1)} \rightarrow 0 \\
0 & \rightarrow M_{-(i+3)} \rightarrow U_{-(i+1)} \rightarrow V_{-(i+1)} \rightarrow 0
\end{aligned}
$$

satisfying the following properties:

$\left(\mathrm{a}_{i}\right)$ the objects $W_{0}, \ldots, W_{-(i+1)}$ are in $\mathcal{W}$;

$\left(\mathrm{b}_{i}\right)$ the sequence $\left(\dagger_{i}\right)$ is $\operatorname{Hom}_{\mathcal{A}}(-, \mathcal{W})$-exact;

$\left(\mathrm{c}_{i}\right)$ one has $U_{-i} \perp \mathcal{W}$ and $U_{-(i+1)} \perp \mathcal{W}$;

$\left(\mathrm{d}_{i}\right)$ one has $V_{-i} \perp \mathcal{W}$ and $V_{-(i+1)} \perp \mathcal{W}$;

$\left(\mathrm{e}_{i}\right)$ one has $V_{-i}$ and $V_{-(i+1)}$ in cores $\widetilde{\mathcal{W}}$.

The sequence $\left(\dagger_{i}\right)$ is obtained by splicing the sequences $\left(\dagger_{0}\right),\left(\circledast_{0}\right), \ldots,\left(\circledast_{i-1}\right)$. Continuing to splice inductively, conditions $\left(\mathrm{a}_{i}\right)-\left(\mathrm{c}_{i}\right)$ show that this process yields a proper $\mathcal{W}$-coresolution of $M$, as desired.

We begin with the base case $i=0$. The membership $G_{-1} \in \operatorname{cores} \widetilde{\mathcal{W}}$ yields a proper $\mathcal{W}$-coresolution of $G_{-1}$ and hence an exact sequence

$$
0 \rightarrow G_{-1} \rightarrow W_{0} \rightarrow V_{0} \rightarrow 0
$$

that is $\operatorname{Hom}_{\mathcal{A}}(-, \mathcal{W})$-exact and with objects $W_{0} \in \mathcal{W}$ and $V_{0} \in \operatorname{cores} \widetilde{\mathcal{W}}$. Using the conditions $G_{-1} \perp \mathcal{W}$ and $W_{0} \perp \mathcal{W}$, Lemma 1.6(a) implies $V_{0} \perp \mathcal{W}$. Consider the pushout diagram whose top row is $\left(\mathbf{w}_{-1}\right)$ and whose middle column is (6).

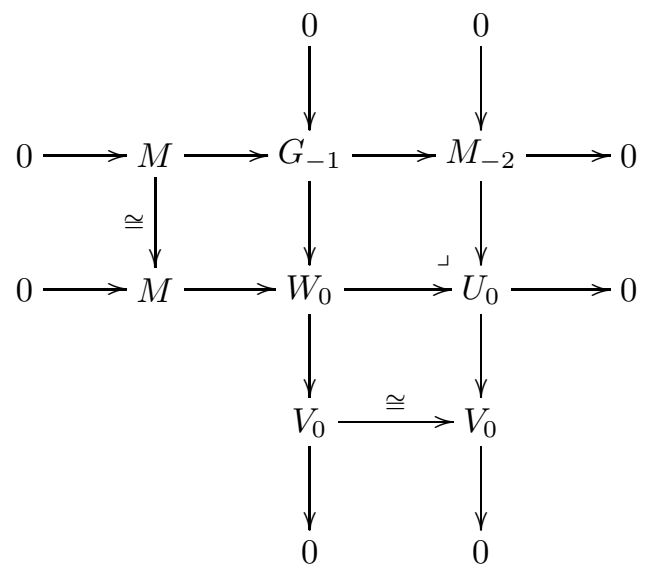

Applying Lemma 1.6(a) to the rightmost column of this diagram, the conditions $M_{-2} \perp \mathcal{W}$ and $V_{0} \perp \mathcal{W}$ imply $U_{0} \perp \mathcal{W}$. For each object $W^{\prime} \in \mathcal{W}$, use the condition $U_{0} \perp W^{\prime}$ with the long exact sequence in $\operatorname{Ext}_{\mathcal{A}}\left(-, W^{\prime}\right)$ asociated to the middle row of this diagram to conclude that this row is $\operatorname{Hom}_{\mathcal{A}}(-, \mathcal{W})$-exact. Set $\left(\dagger_{0}\right)$ equal to the middle row of (7), and set $\left(*_{0}\right)$ equal to the rightmost column of (7). Construct the next pushout diagram using $\left(\mathbf{w}_{-2}\right)$ in the top row and the rightmost column 
of (7) in the left column.

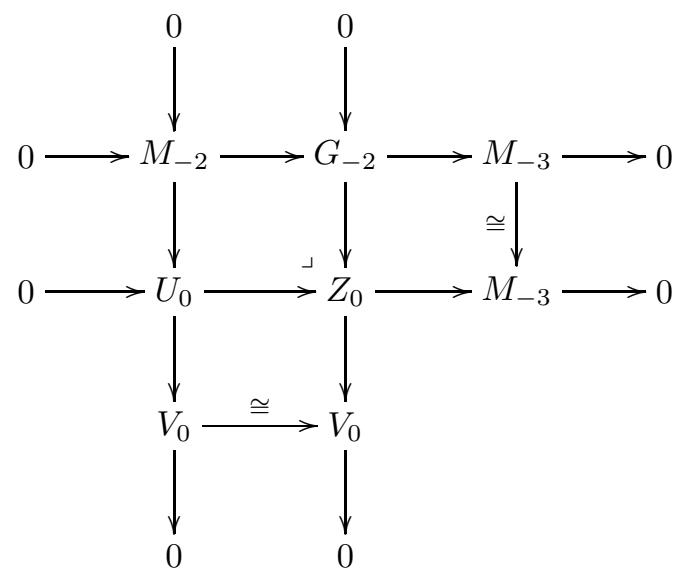

As in the above discussion, the condition $V_{0} \perp \mathcal{W}$ implies that the middle column of (8) is $\operatorname{Hom}_{\mathcal{A}}(-\mathcal{W})$-exact. Using Lemma 1.6(a) with this column, the conditions $G_{-2} \perp \mathcal{W}$ and $V_{0} \perp \mathcal{W}$ yield $Z_{0} \perp \mathcal{W}$. We know that $G_{-2}$ and $V_{0}$ are in cores $\widetilde{\mathcal{W}}$, so an application of Lemma $1.9(\mathrm{~b})$ to this column implies $Z_{0} \in$ cores $\widetilde{\mathcal{W}}$. A proper $\mathcal{W}$-coresolution of $Z_{0}$ provides an exact sequence

$$
0 \rightarrow Z_{0} \rightarrow W_{-1} \rightarrow V_{-1} \rightarrow 0
$$

that is $\operatorname{Hom}_{\mathcal{A}}(-, \mathcal{W})$-exact and with objects $W_{-1} \in \mathcal{W}$ and $V_{-1} \in \operatorname{cores} \widetilde{\mathcal{W}}$. Again using Lemma 1.6(a), the conditions $Z_{0} \perp \mathcal{W}$ and $W_{-1} \perp \mathcal{W}$ conspire with the $\operatorname{Hom}_{\mathcal{A}}(-, \mathcal{W})$-exactness of (9) to imply $V_{-1} \perp \mathcal{W}$. Consider the next pushout diagram whose top row is the middle row of (8) and whose middle column is (9).

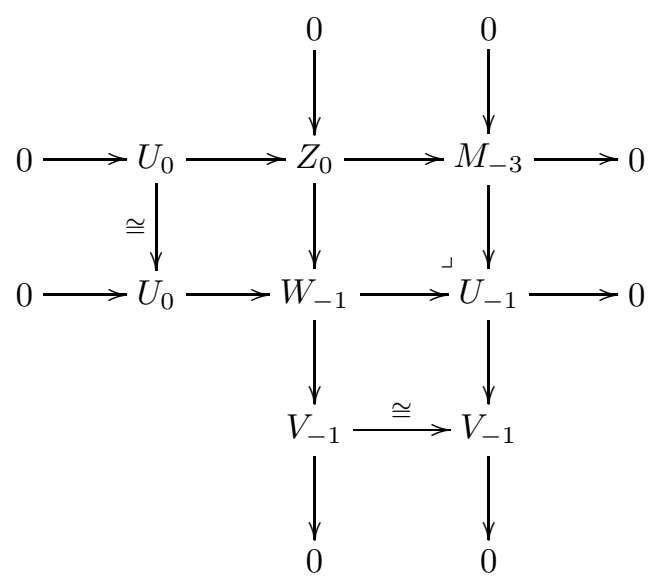

Set $\left(\circledast_{0}\right)$ equal to the middle row of this diagram, and $\left(\ddagger_{0}\right)$ equal to the rightmost column. Thanks to Lemma 1.6(a), the conditions $M_{-3} \perp \mathcal{W}$ and $V_{-1} \perp \mathcal{W}$ imply $U_{-1} \perp \mathcal{W}$. Thus, the conditions $\left(\mathrm{a}_{0}\right)-\left(\mathrm{e}_{0}\right)$ are satisfied, establishing the base case.

For the induction step, assume that the exact sequences $\left(\dagger_{i}\right),\left(*_{i}\right),\left(\circledast_{i}\right)$, and $\left(\ddagger_{i}\right)$ have been constructed satisfying the conditions $\left(\mathrm{a}_{i}\right)-\left(\mathrm{e}_{i}\right)$. Note that condition $\left(\mathrm{c}_{i}\right)$ implies that the sequence $\left(\circledast_{i}\right)$ is $\operatorname{Hom}_{\mathcal{A}}(-, \mathcal{W})$-exact. Thus, we may splice together 
the sequences $\left(\dagger_{i}\right)$ and $\left(\circledast_{i}\right)$ to construct the sequence $\left(\dagger_{i+1}\right)$ which is exact and $\operatorname{Hom}_{\mathcal{A}}(-, \mathcal{W})$-exact and such that $W_{0}, \ldots, W_{-(i+1)} \in \mathcal{W}$. Also, set $\left(*_{i+1}\right)=\left(\ddagger_{i}\right)$.

The next pushout diagram has $\left(\mathbf{W}_{i+3}\right)$ in the top row and $\left(\ddagger_{i}\right)$ in the left column.

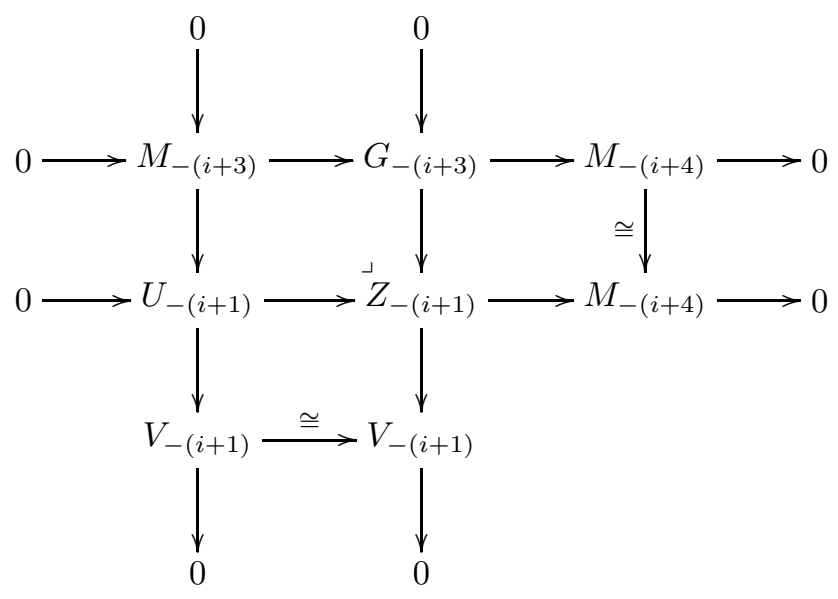

With the long exact sequence in $\operatorname{Ext}_{\mathcal{A}}(-,-)$, the condition $V_{-(i+1)} \perp \mathcal{W}$ implies that the middle column of (11) is $\operatorname{Hom}_{\mathcal{A}}(-, \mathcal{W})$-exact. Using Lemma 1.6(可) with this column, the conditions $G_{-(i+3)} \perp \mathcal{W}$ and $V_{-(i+1)} \perp \mathcal{W}$ yield $Z_{-(i+1)} \perp \mathcal{W}$. As $G_{-(i+3)}$ and $V_{-(i+1)}$ are in cores $\widetilde{\mathcal{W}}$, apply Lemma $1.9(\mathrm{~b})$ to this column to conclude $Z_{-(i+1)} \in \operatorname{cores} \widetilde{\mathcal{W}}$. A proper $\mathcal{W}$-coresolution of $Z_{-(i+1)}$ provides an exact sequence

$$
0 \rightarrow Z_{-(i+1)} \rightarrow W_{-(i+2)} \rightarrow V_{-(i+2)} \rightarrow 0
$$

that is $\operatorname{Hom}_{\mathcal{A}}(-, \mathcal{W})$-exact and with objects $W_{-(i+2)} \in \mathcal{W}$ and $V_{-(i+2)} \in \operatorname{cores} \widetilde{\mathcal{W}}$. Again using Lemma 1.6(国) and the $\operatorname{Hom}_{\mathcal{A}}(-, \mathcal{W})$-exactness of (12), the conditions $Z_{-(i+1)} \perp \mathcal{W}$ and $W_{-(i+2)} \perp \mathcal{W}$ imply $V_{-(i+2)} \perp \mathcal{W}$. Consider the next pushout diagram whose top row is the middle row of (11) and whose middle column is (12).

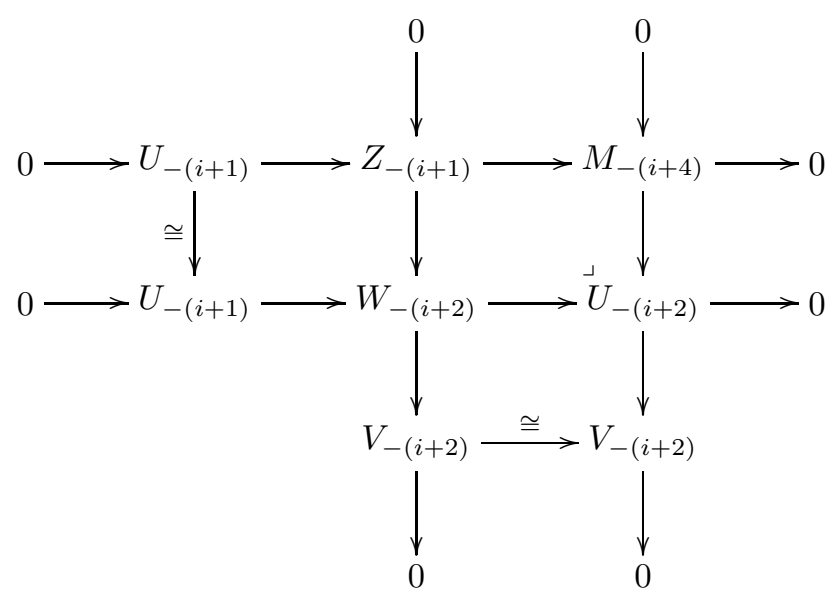

Set $\left(\circledast_{i+1}\right)$ equal to the middle row of this diagram, and set $\left(\ddagger_{i+1}\right)$ equal to the rightmost column. With Lemma 1.6(国), the conditions $M_{-(i+4)} \perp \mathcal{W}$ and $V_{-(i+2)} \perp \mathcal{W}$ imply $U_{-(i+2)} \perp \mathcal{W}$. Thus, the conditions $\left(\mathrm{a}_{i+1}\right)-\left(\mathrm{e}_{i+1}\right)$ are satisfied, establishing the induction step. 
What follows is the second step in the proof of Theorem $\mathrm{A}$ from the introduction. See Example 5.9 for the necessity of the cogeneration hypothesis.

Theorem 4.9. If $\mathcal{X}$ is closed under extensions and $\mathcal{W}$ is both an injective cogenerator and a projective generator for $\mathcal{X}$, then $\mathcal{G}^{n}(\mathcal{X}) \subseteq \mathcal{G}(\mathcal{W})$ for each $n \geqslant 1$.

Proof. Let $N$ be an object in $\mathcal{G}^{n}(\mathcal{X})$. By Theorem 4.8 we know that $N$ admits a proper $\mathcal{W}$-resolution $W^{\prime} \stackrel{\simeq}{\rightarrow} N$ and a proper $\mathcal{W}$-coresolution $N \stackrel{\simeq}{\rightarrow} W^{\prime \prime}$. We will show that ${ }^{+} W^{\prime}$ is $\operatorname{Hom}_{\mathcal{A}}(-, \mathcal{W})$-exact and that ${ }^{+} W^{\prime \prime}$ is $\operatorname{Hom}_{\mathcal{A}}(\mathcal{W},-)$-exact. Since we already know that ${ }^{+} W^{\prime}$ is $\operatorname{Hom}_{\mathcal{A}}(\mathcal{W},-)$-exact and that ${ }^{+} W^{\prime \prime}$ is $\operatorname{Hom}_{\mathcal{A}}(-, \mathcal{W})$ exact, this will show that the concatenated complex

$$
\cdots \rightarrow W_{1}^{\prime} \rightarrow W_{0}^{\prime} \rightarrow W_{0}^{\prime \prime} \rightarrow W_{-1}^{\prime} \rightarrow \cdots
$$

is a complete $\mathcal{W}$-resolution of $N$, completing the proof.

We will show that ${ }^{+} W^{\prime}$ is $\operatorname{Hom}_{\mathcal{A}}(-, \mathcal{W})$-exact; the proof of the other fact is dual. For each $i \geqslant 0$, there is an exact sequence

$$
0 \rightarrow N_{i+1} \rightarrow W_{i}^{\prime} \rightarrow N_{i} \rightarrow 0
$$

We have $N_{0}=N$ and so $N_{0} \perp \mathcal{W}$ is true by Proposition 4.3 and $W_{i}^{\prime} \perp \mathcal{W}$ by assumption. Using Lemma 1.6(a) , an induction argument implies $N_{i} \perp \mathcal{W}$ for each $i$. It follows that $\left(*_{i}\right)$ is $\operatorname{Hom}_{\mathcal{A}}(-, \mathcal{W})$-exact, and it follows that ${ }^{+} W^{\prime}$ is $\operatorname{Hom}_{\mathcal{A}}(-, \mathcal{W})$-exact, as desired.

Theorem $\mathrm{A}$ from the introduction follows from the next result; see Example 5.3 .

Corollary 4.10. If $\mathcal{W} \perp \mathcal{W}$, then $\mathcal{G}^{n}(\mathcal{W})=\mathcal{G}(\mathcal{W})$ for each $n \geqslant 1$.

Proof. Note that Corollaries 4.5 and 4.7 imply that $\mathcal{G}(\mathcal{W})$ is closed under extensions and that $\mathcal{W}$ is both an injective cogenerator and a projective generator for $\mathcal{G}(\mathcal{W})$.

We argue by induction on $n$, the case $n=1$ being trivial. For $n>1$, if $\mathcal{G}^{n-1}(\mathcal{W})=\mathcal{G}(\mathcal{W})$, then setting $\mathcal{X}=\mathcal{G}(\mathcal{W})$ in Theorem 4.9 yields the final containment in the next sequence

$$
\mathcal{G}(\mathcal{W}) \subseteq \mathcal{G}^{n}(\mathcal{W})=\mathcal{G}\left(\mathcal{G}^{n-1}(\mathcal{W})\right)=\mathcal{G}(\mathcal{G}(\mathcal{W})) \subseteq \mathcal{G}(\mathcal{W})
$$

and hence the desired conclusion.

With Corollary 4.5, the final two results of this section contain Theorem B from the introduction; compare to [6. (4.3.5)]

Proposition 4.11. If $\mathcal{W} \perp \mathcal{W}$, then $\mathcal{G}(\mathcal{W})$ is closed under direct summands.

Proof. Let $A^{\prime}$ and $A^{\prime \prime}$ be objects in $\mathcal{A}$ such that $A^{\prime} \oplus A^{\prime \prime}$ is in $\mathcal{G}(\mathcal{W})$. We construct proper $\mathcal{W}$-resolutions $W^{\prime} \stackrel{\simeq}{\rightarrow} A^{\prime}$ and $W^{\prime \prime} \stackrel{\simeq}{\rightarrow} A^{\prime \prime}$ such that $\left(W^{\prime}\right)^{+}$and $\left(W^{\prime \prime}\right)^{+}$are $\operatorname{Hom}_{\mathcal{A}}(-, \mathcal{W})$-exact. Dually, one constructs proper $\mathcal{W}$-coresolutions $A^{\prime} \stackrel{\simeq}{\rightarrow} V^{\prime}$ and $A^{\prime \prime} \stackrel{\simeq}{\rightarrow} V^{\prime \prime}$ such that ${ }^{+} V^{\prime}$ and ${ }^{+} V^{\prime \prime}$ are $\operatorname{Hom}_{\mathcal{A}}(\mathcal{W},-)$-exact, and this shows that $A^{\prime}$ and $A^{\prime \prime}$ are in $\mathcal{G}(\mathcal{W})$.

Observe first that $A^{\prime}$ and $A^{\prime \prime}$ both admit (augmented) proper $\mathcal{G}(\mathcal{W})$-resolutions

$$
\begin{aligned}
& X^{\prime}=\cdots \stackrel{\left(\begin{array}{cc}
\text { id } & 0 \\
0 & 0
\end{array}\right)}{\longrightarrow} A^{\prime} \oplus A^{\prime \prime} \stackrel{\left(\begin{array}{ll}
0 & 0 \\
0 & \text { id }
\end{array}\right)}{\longrightarrow} A^{\prime} \oplus A^{\prime \prime} \stackrel{(\text { id }}{\longrightarrow} A^{\prime} \rightarrow 0 \\
& X^{\prime \prime}=\cdots \stackrel{\left(\begin{array}{ll}
0 & 0 \\
0 & \text { id }
\end{array}\right)}{\longrightarrow} A^{\prime} \oplus A^{\prime \prime} \stackrel{\left(\begin{array}{cc}
\text { id } & 0 \\
0 & 0
\end{array}\right)}{\longrightarrow} A^{\prime} \oplus A^{\prime \prime} \stackrel{(0 \text { id })}{\longrightarrow} A^{\prime \prime} \rightarrow 0
\end{aligned}
$$

where properness follows from the contractibility of $X^{\prime}$ and $X^{\prime \prime}$. From Proposition 4.3 we know $\mathcal{W} \perp\left(A^{\prime} \oplus A^{\prime \prime}\right)$, so the additivity of Ext ${ }_{\mathcal{A}}$ implies $\mathcal{W} \perp A^{\prime}$ 
and $\mathcal{W} \perp A^{\prime \prime}$. Lemma $2.2(\underline{\underline{b}})$ and Corollary 4.7 imply that $A^{\prime}$ and $A^{\prime \prime}$ admit proper $\mathcal{W}$-resolutions $W^{\prime} \stackrel{\simeq}{\rightarrow} A^{\prime}$ and $W^{\prime \prime} \stackrel{\simeq}{\rightarrow} A^{\prime \prime}$, so $W^{\prime} \oplus W^{\prime \prime} \stackrel{\simeq}{\rightarrow} A^{\prime} \oplus A^{\prime \prime}$ is a proper $\mathcal{W}$-resolution. We show that $\left(W^{\prime}\right)^{+}$and $\left(W^{\prime \prime}\right)^{+}$are $\operatorname{Hom}_{\mathcal{A}}(-, \mathcal{W})$-exact. As $A^{\prime} \oplus A^{\prime \prime}$ is in $\mathcal{G}(\mathcal{W})$, it admits a proper $\mathcal{W}$-resolution $W \stackrel{\simeq}{\rightarrow} A^{\prime} \oplus A^{\prime \prime}$ such that $W^{+}$is $\operatorname{Hom}_{\mathcal{A}}(-, \mathcal{W})$-exact. Hence, the resolutions $W$ and $W^{\prime} \oplus W^{\prime \prime}$ are homotopy equivalent. Because $W^{+}$is $\operatorname{Hom}_{\mathcal{A}}(-, \mathcal{W})$-exact, we know that $\left(W^{\prime} \oplus W^{\prime \prime}\right)^{+}$is also $\operatorname{Hom}_{\mathcal{A}}(-, \mathcal{W})$-exact, and so $\left(W^{\prime}\right)^{+}$and $\left(W^{\prime \prime}\right)^{+}$are $\operatorname{Hom}_{\mathcal{A}}(-, \mathcal{W})$-exact.

Theorem 4.12. Assume $\mathcal{W} \perp \mathcal{W}$.

(a) If $\mathcal{W}$ is closed under kernels of epimorphisms, then so is $\mathcal{G}(\mathcal{W})$.

(b) If $\mathcal{W}$ is closed under cokernels of monomorphisms, then so is $\mathcal{G}(\mathcal{W})$.

Proof. We prove part (国); the proof of part (B) is dual. Consider an exact sequence in $\mathcal{A}$ with objects $N$ and $N^{\prime \prime}$ in $\mathcal{G}(\mathcal{W})$.

$$
0 \rightarrow N^{\prime} \rightarrow N \stackrel{\tau}{\rightarrow} N^{\prime \prime} \rightarrow 0
$$

Let $W$ and $W^{\prime \prime}$ be complete $\mathcal{W}$-resolutions of $N$ and $N^{\prime \prime}$, respectively.

We first construct a commutative diagram of the following form

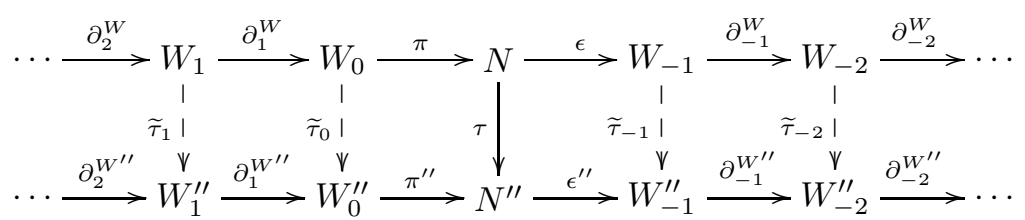

where $\epsilon \pi=\partial_{0}^{W}$ and $\epsilon^{\prime \prime} \pi^{\prime \prime}=\partial_{0}^{W^{\prime \prime}}$. Since $\left(W_{\geqslant 0}\right)^{+}$is a chain complex and $\left(W_{\geqslant 0}^{\prime \prime}\right)^{+}$is $\operatorname{Hom}_{\mathcal{A}}(\mathcal{W},-)$ exact, one can successively lift $\tau$ to the left as in the diagram; argue as in [14, (1.8)]. Dually, since ${ }^{+} W_{<0}^{\prime \prime}$ is a chain complex and ${ }^{+} W_{<0}$ is $\operatorname{Hom}_{\mathcal{A}}(-, \mathcal{W})$ exact, one can successively lift $\tau$ to the right as in the diagram.

Thus, we have constructed a morphism of chain complexes $\widetilde{\tau}: W \rightarrow W^{\prime \prime}$ such that the induced map $\operatorname{Coker}\left(\partial_{1}^{W}\right) \rightarrow \operatorname{Coker}\left(\partial_{1}^{W^{\prime \prime}}\right)$ is equivalent to $\tau$.

Next, we show that there exists a complex $\widetilde{W}$ with and a morphism $\tau^{\prime}: W \oplus \widetilde{W} \rightarrow$ $W^{\prime \prime}$ satisfying the following properties:

(a) $\widetilde{W}$ is contractible and $\widetilde{W}_{n}$ is in $\mathcal{W}$ for each $n \in \mathbb{Z}$.

(b) $\tau_{n}^{\prime}$ is an epimorphism for each $n \in \mathbb{Z}$.

(c) The natural monomorphism $W \stackrel{\epsilon}{\rightarrow} W \oplus \widetilde{W}$ satisfies $\widetilde{\tau}=\tau^{\prime} \epsilon$.

The complex $\widetilde{W}=\Sigma^{-1}$ Cone $\left(\operatorname{id}_{W^{\prime \prime}}\right)$ is contractible; see 1.3 Let $f: \widetilde{W} \rightarrow W^{\prime \prime}$ denote the composition of the natural morphisms $\widetilde{W}=\Sigma^{-1}$ Cone $\left(\operatorname{id}_{W^{\prime \prime}}\right) \rightarrow W^{\prime \prime}$. Note that each $f_{n}$ is a split epimorphism. It follows that the homomorphisms $\tau_{n}^{\prime}=\left(\widetilde{\tau}_{n} f_{n}\right): W_{n} \oplus \widetilde{W}_{n} \rightarrow W_{n}^{\prime \prime}$ describe a morphism of complexes satisfying the desired properties.

Because of property (国) the complex $\widetilde{W}$ is a complete $\mathcal{W}$-resolution; see Remark 4.2, Set $\widetilde{N}=\operatorname{Coker}\left(\partial_{1}^{\widetilde{W}}\right)$, which is an object in $\mathcal{G}(\mathcal{W})$ with complete resolution $\widetilde{W}$. Furthermore, one has $\operatorname{Coker}\left(\partial_{1}^{W \oplus \widetilde{W}}\right) \cong N \oplus \widetilde{N}$, and the morphism $\tau^{\prime}$ induces a homomorphism $N \oplus \widetilde{N} \stackrel{f=(\tau \pi)}{\longrightarrow} N^{\prime \prime}$. Because $\tau$ is surjective, the map $f$ is also surjective. We will show that $\operatorname{Ker}(f)$ is in $\mathcal{G}(\mathcal{W})$, and then we will show that $N^{\prime}=\operatorname{Ker}(\tau)$ is in $\mathcal{G}(\mathcal{W})$. 
The morphism $\tau^{\prime}$ is degreewise surjective. As $\mathcal{W}$ is closed under kernels of epimorphisms, it follows that the complex $W^{\prime}=\operatorname{Ker}(\widetilde{\tau})$ consists of objects in $\mathcal{W}$. The next exact sequence shows that $W^{\prime}$ is exact because $W, \widetilde{W}$, and $W^{\prime \prime}$ are so

$$
0 \rightarrow W^{\prime} \rightarrow W \oplus \widetilde{W} \stackrel{\tau^{\prime}}{\rightarrow} W^{\prime \prime} \rightarrow 0
$$

This sequence induces a second exact sequence

$$
0 \rightarrow W_{\geqslant 0}^{\prime} \rightarrow W_{\geqslant 0} \oplus \widetilde{W}_{\geqslant 0} \rightarrow W_{\geqslant 0}^{\prime \prime} \rightarrow 0
$$

whose associated long exact sequence is of the form

$$
0 \rightarrow \operatorname{Coker}\left(\partial_{1}^{W^{\prime}}\right) \rightarrow N \stackrel{f}{\rightarrow} N^{\prime \prime} \rightarrow 0 .
$$

Thus, we have $\operatorname{Ker}(f) \cong \operatorname{Coker}\left(\partial_{1}^{W}\right)$. To show that $\operatorname{Ker}(f)$ is in $\mathcal{G}(\mathcal{W})$, it suffices to show that $W^{\prime}$ is $\operatorname{Hom}_{\mathcal{A}}(\mathcal{W},-)$-exact and $\operatorname{Hom}_{\mathcal{A}}(-, \mathcal{W})$-exact. For each object $U$ in $\mathcal{W}$, the next sequence of complexes is exact as $\mathcal{W} \perp \mathcal{W}$

$$
0 \rightarrow \operatorname{Hom}_{\mathcal{A}}\left(U, W^{\prime}\right) \rightarrow \operatorname{Hom}_{\mathcal{A}}(U, W) \rightarrow \operatorname{Hom}_{\mathcal{A}}\left(U, W^{\prime \prime}\right) \rightarrow 0
$$

Since $W$ and $W^{\prime \prime}$ are $\operatorname{Hom}_{\mathcal{A}}(\mathcal{W},-)$-exact, the associated long exact sequence shows that $W^{\prime}$ is also $\operatorname{Hom}_{\mathcal{A}}(\mathcal{W},-)$-exact. Dually, one shows that $W^{\prime}$ is $\operatorname{Hom}_{\mathcal{A}}(-, \mathcal{W})$ exact, thus showing that $\operatorname{Ker}(f)$ is in $\mathcal{G}(\mathcal{W})$.

To see that $N^{\prime}$ is in $\mathcal{G}(\mathcal{W})$, consider the following pullback diagram whose rightmost column is (14) and whose middle row is the natural split exact sequence.

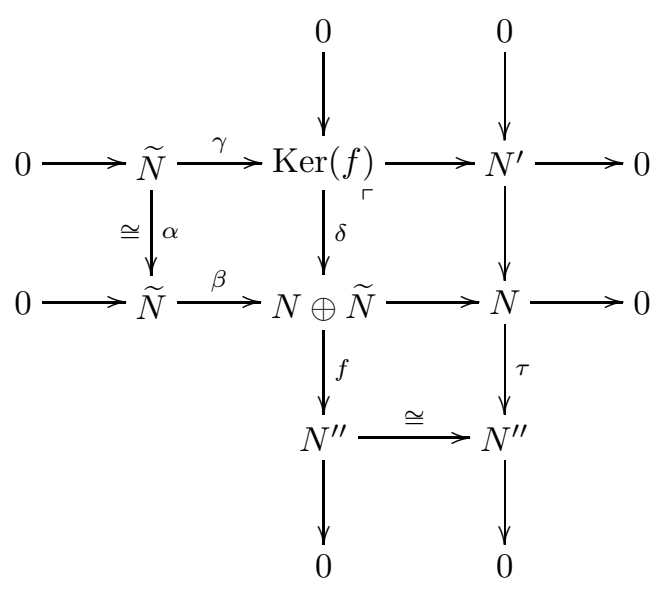

Let $\sigma: N \oplus \widetilde{N} \rightarrow \widetilde{N}$ denote the natural surjection. It follows that $\sigma \beta=\mathrm{id}_{\widetilde{N}}$. Since $\alpha$ is an isomorphism, the equality $\delta \gamma=\beta \alpha$ implies

$$
\left(\alpha^{-1} \sigma \delta\right) \gamma=\alpha^{-1} \sigma \beta \alpha=\alpha^{-1} \alpha=\operatorname{id}_{\widetilde{N}}
$$

and so the top row of (16) is split exact. Hence, the object $N^{\prime}$ is a direct summand of $\operatorname{Ker}(f)$. We have shown that $\operatorname{Ker}(f)$ is in $\mathcal{G}(\mathcal{W})$. The category $\mathcal{G}(\mathcal{W})$ is closed under direct summands by Proposition 4.11, and so $N^{\prime}$ is in $\mathcal{G}(\mathcal{W})$ as desired.

\section{Consequences for CATEgories of interest}

Let $R$ be a commutative ring and $C$ a semidualizing $R$-module. We now apply the results of Section 4 to the examples in Section 3 . We begin with some computations.

Example 5.1. The relevant definitions yield equalities $\mathcal{G}(\mathcal{P}(R))=\mathcal{G P}(R)$ and $\mathcal{G}(\mathcal{I}(R))=\mathcal{G I}(R)$. If $R$ is noetherian, then $\mathcal{G}\left(\mathcal{P}^{f}(R)\right)=\mathcal{G}(R)$. 
The next result generalizes the previous example.

Proposition 5.2. Let $R$ be a commutative ring. If $C$ is $R$-semidualizing, then $\mathcal{G}\left(\mathcal{P}_{C}(R)\right)=\mathcal{G} \mathcal{P}_{C}(R) \cap \mathcal{B}_{C}(R)$ and $\mathcal{G}\left(\mathcal{I}_{C}(R)\right)=\mathcal{G I}_{C}(R) \cap \mathcal{A}_{C}(R)$. If further $R$ is noetherian, then $\mathcal{G}\left(\mathcal{P}_{C}^{f}(R)\right)=\mathcal{G}_{C}(R) \cap \mathcal{B}_{C}(R)$.

Proof. We will prove the first equality; the others are proved similarly. For one containment, let $M$ be an object in $\mathcal{G}\left(\mathcal{P}_{C}(R)\right)$. To show that $M$ is an object in $\mathcal{G} \mathcal{P}_{C}(R)$, we use [20, $\left.(3.2)\right]$ : it suffices to show that $M$ admits a proper $\mathcal{P}_{C}(R)$ coresolution and $M \perp \mathcal{P}_{C}(R)$. The first of these is in Remark4.2 which says that $M$ is in cores $\widehat{\mathcal{P}_{C}(R)}$; the second one is in Proposition 4.3 which implies $\mathcal{G}\left(\mathcal{P}_{C}\right) \perp \mathcal{P}_{C}$. To show that $M$ is an object in $\mathcal{B}_{C}(R)$, we need to verify $\operatorname{Ext}_{R}^{\geqslant 1}(C, M)=0$ and $\operatorname{Tor}_{\geqslant 1}^{R}\left(C, \operatorname{Hom}_{R}(C, M)\right)=0$ and $M \cong C \otimes_{R} \operatorname{Hom}_{R}(C, M)$. The first of these is in Proposition 4.3 which implies $\mathcal{P}_{C} \perp \mathcal{G}\left(\mathcal{P}_{C}\right)$, and the others are in [18, (2.2)].

For the reverse containment, fix an object $N$ in $\mathcal{G} \mathcal{P}_{C}(R) \cap \mathcal{B}_{C}(R)$. Since $N$ is in $\mathcal{G} \mathcal{P}_{C}(R)$, it admits a complete $\mathcal{P} \mathcal{P}_{C}$-resolution $Y$, so the complex $Y_{<0}$ is a proper $\mathcal{P}_{C}(R)$-coresolution of $N$. Also, $N$ admits a proper $\mathcal{P}_{C}(R)$-resolution $Z$ by 18 , (2.4)] as $N$ is in $\mathcal{B}_{C}(R)$. Once it is shown that $Y_{<0}^{+}$is $\operatorname{Hom}_{R}\left(\mathcal{P}_{C}(R),-\right)$-exact and $Z^{+}$is $\operatorname{Hom}_{R}\left(-, \mathcal{P}_{C}(R)\right)$-exact, a complete $\mathcal{P} \mathcal{P}_{C}$-resolution of $N$ will be obtained by splicing $Z$ and $Y_{<0}$.

To see that $Y_{<0}^{+}$is $\operatorname{Hom}_{R}\left(\mathcal{P}_{C}(R),-\right)$-exact, set $N^{(0)}=N$ and $N^{(i)}=\operatorname{Coker}\left(\partial_{i-1}^{Y}\right)$ for each $i \leqslant-1$. From [16, (5.2)], we know that $Y_{i}$ is in $\mathcal{B}_{C}(R)$ for each $i \leqslant-1$. Since $N$ is also in $\mathcal{B}_{C}(R)$, an induction argument using the exact sequence

$$
0 \rightarrow N^{(i-1)} \rightarrow Y_{i} \rightarrow N^{(i)} \rightarrow 0
$$

implies that $N^{(i)}$ is in $\mathcal{B}_{C}(R)$ for each $i \leqslant-1$. For each projective $R$-module $P$, this yields the vanishing in the next sequence

$$
\operatorname{Ext}_{R}^{1}\left(P \otimes_{R} C, N^{(i)}\right) \cong \operatorname{Hom}_{R}\left(P, \operatorname{Ext}_{R}^{1}\left(C, N^{(i)}\right)\right)=0
$$

while the isomorphism is from Hom-Tensor adjunction. It follows that each sequence $\left(*_{i}\right)$ is $\operatorname{Hom}_{R}\left(\mathcal{P}_{C}(R),-\right)$-exact, and thus so is $Y_{<0}^{+}$.

To see that $Z^{+}$is $\operatorname{Hom}_{R}\left(-, \mathcal{P}_{C}(R)\right)$-exact, it suffices to let $P$ be projective and to justify the following sequence for $i \geqslant 1$.

$$
\mathrm{H}_{-i}\left(\operatorname{Hom}_{R}\left(Z^{+}, P \otimes_{R} C\right)\right)=\mathrm{H}_{-i}\left(\operatorname{Hom}_{R}\left(Z, P \otimes_{R} C\right)\right) \cong \operatorname{Ext}_{R}^{i}\left(N, P \otimes_{R} C\right)=0
$$

The isomorphism is from [18, (4.2.a)] because $N$ and $P \otimes_{R} C$ are in $\mathcal{B}_{C}(R)$. The vanishing follows because $N$ is in $\mathcal{G P} \mathcal{P}_{C}(R)$ and $\mathcal{G} \mathcal{P}_{C}(R) \perp \mathcal{P}_{C}(R)$; see [20, (3.2)].

We now outline the consequences of Corollaries 4.7 and 4.10 for the examples of Section 3. The first example below contains Theorem $\mathrm{A}$ from the introduction.

Example 5.3. The category $\mathcal{P}_{C}(R)$ is an injective cogenerator and a projective generator for $\mathcal{G} \mathcal{P}_{C}(R) \cap \mathcal{B}_{C}(R)$, and $\mathcal{G}^{n}\left(\mathcal{P}_{C}(R)\right)=\mathcal{G} \mathcal{P}_{C}(R) \cap \mathcal{B}_{C}(R)$ for each $n \geqslant 1$. Hence, $\mathcal{P}(R)$ is an injective cogenerator and a projective generator for $\mathcal{G} \mathcal{P}(R)$, and $\mathcal{G}^{n}(\mathcal{P}(R))=\mathcal{G P}(R)$. If $\mathcal{A}$ has enough projectives, then $\mathcal{P}(\mathcal{A})$ is an injective cogenerator and a projective generator for $\mathcal{G}(\mathcal{P}(\mathcal{A}))$, and $\mathcal{G}^{n}(\mathcal{P}(\mathcal{A}))=\mathcal{G}(\mathcal{P}(\mathcal{A}))$.

Example 5.4. The category $\mathcal{I}_{C}(R)$ is an injective cogenerator and a projective generator for $\mathcal{G I}_{C}(R) \cap \mathcal{A}_{C}(R)$, and $\mathcal{G}^{n}\left(\mathcal{I}_{C}(R)\right)=\mathcal{G} \mathcal{I}_{C}(R) \cap \mathcal{A}_{C}(R)$ for each $n \geqslant$ 1. Hence, $\mathcal{I}(R)$ is an injective cogenerator and a projective generator for $\mathcal{G} \mathcal{I}(R)$, and $\mathcal{G}^{n}(\mathcal{I}(R))=\mathcal{G} \mathcal{I}(R)$. If $\mathcal{A}$ has enough injectives, then $\mathcal{I}(\mathcal{A})$ is an injective cogenerator and a projective generator for $\mathcal{G}(\mathcal{I}(\mathcal{A}))$, and $\mathcal{G}^{n}(\mathcal{I}(\mathcal{A}))=\mathcal{G}(\mathcal{I}(\mathcal{A}))$. 
Example 5.5. Assume that $R$ is noetherian. Then $\mathcal{P}_{C}^{f}(R)$ is an injective cogenerator and a projective generator for $\mathcal{G}_{C}(R) \cap \mathcal{B}_{C}(R)$, and $\mathcal{G}^{n}\left(\mathcal{P}_{C}^{f}(R)\right)=\mathcal{G}_{C}(R) \cap \mathcal{B}_{C}(R)$. In particular, $\mathcal{P}^{f}(R)$ is an injective cogenerator and a projective generator for $\mathcal{G}(R)$, and $\mathcal{G}^{n}\left(\mathcal{P}^{f}(R)\right)=\mathcal{G}(R)$.

Example 5.6. The category $\mathcal{F}^{\prime}(R)$ is an injective cogenerator and a projective generator for $\mathcal{G}\left(\mathcal{F}^{\prime}(R)\right)$, and $\mathcal{G}^{n}\left(\mathcal{F}^{\prime}(R)\right)=\mathcal{G}\left(\mathcal{F}^{\prime}(R)\right)$ for each $n \geqslant 1$.

With Proposition 4.3 and Corollary 4.5 in mind, we now show that $\mathcal{W} \perp \mathcal{W}$ need not imply $\mathcal{G}(\mathcal{W}) \perp \mathcal{G}(\mathcal{W})$.

Example 5.7. Let $(R, \mathfrak{m}, k)$ be a local, nonregular, Gorenstein, artinian ring. With $\mathcal{W}=\mathcal{P}^{f}(R)$, we have $\mathcal{G}(\mathcal{W})=\mathcal{G}(R)=\mathcal{M}^{f}(R)$ where the last equality is because $R$ is artinian and Gorenstein; see [6, (1.4.8),(1.4.9)]. We know $\operatorname{Ext}_{R}^{\geqslant 1}(k, k) \neq 0$ since $R$ is nonregular, and so $\mathcal{G}(\mathcal{W}) \not \perp \mathcal{G}(\mathcal{W})$.

We conclude with some questions and final observations.

Question 5.8. Must there be an equality $\mathcal{G}^{n}(\mathcal{X})=\mathcal{G}(\mathcal{X})$ for each $n \geqslant 1$ ? Is $\mathcal{G}(\mathcal{X})$ always exact? Is $\mathcal{G}(\mathcal{X})$ always closed under kernels of epimorphisms or cokernels of monomorphisms? Must $\mathcal{G}(\mathcal{W})$ be contained in $\mathcal{G}(\mathcal{X})$ ? Can $\mathcal{G}(\mathcal{F}(R))$ or $\mathcal{G}\left(\mathcal{F}^{\prime}(R)\right)$ or $\mathcal{G}\left(\mathcal{G}^{\prime}(R)\right)$ be identified as in Proposition 5.2.

The final examples are presented with an eye toward the last question in 5.8

Example 5.9. If $(R, \mathfrak{m})$ is a noetherian local ring and $\operatorname{dim}(R) \geqslant 1$, then $\mathcal{G}(\mathcal{F}(R)) \nsubseteq$ $\mathcal{G}(\mathcal{P}(R))$. Indeed, the ring of formal power series $R \llbracket X \rrbracket$ is a flat $R$-module, so it is in $\mathcal{G}(\mathcal{F}(R))$. Suppose by way of contradiction that $R \llbracket X \rrbracket$ is in $\mathcal{G}(\mathcal{P}(R))$. First note that [17, Prop. 6] yields $\operatorname{pd}_{R}(R \llbracket X \rrbracket)<\infty$, and so [14, (2.7)] implies $\operatorname{pd}_{R}(R \llbracket X \rrbracket)=$ $\mathcal{G}-\operatorname{pd}_{R}(R \llbracket X \rrbracket)=0$. It follows that $R \llbracket X \rrbracket$ is projective, contradicting [5, (2.1)].

From this it follows that the conclusion of Theorem 4.9 need not hold if $\mathcal{W}$ is not a cogenerator for $\mathcal{X}$. To see this, assume that $R$ is $\mathfrak{m}$-adically complete. Standard results combine to show that $\mathcal{P}(R)$ is a projective generator for $\mathcal{F}(R)$ and that $\mathcal{F}(R)$ is closed under extensions. Furthermore, one has $\mathcal{F}(R) \perp \mathcal{P}(R)$ by [9, (5.3.28)].

With Theorem 4.9, the previous example provides the next result.

Corollary 5.10. If $R$ is a complete local notherian ring and $\operatorname{dim}(R) \geqslant 1$, then $\mathcal{P}(R)$ is not a cogenerator for $\mathcal{F}(R)$.

Example 5.11. Let $(R, \mathfrak{m})$ be a noetherian local ring. If $R$ is not $\mathfrak{m}$-adically complete, then $\mathcal{G}\left(\mathcal{F}^{\prime}(R)\right) \nsubseteq \subseteq \mathcal{G}(\mathcal{P}(R))$. The $\mathfrak{m}$-adic completion $\widehat{R}$ is flat and cotorsion; see, e.g., [9, (5.3.28)]. Arguing as in Example [5.9, it then suffices to note that $\widehat{R}$ is not projective by [11, Thm. A].

Example 5.12. Assume that $R$ is local and noetherian. Using Example 5.3 and [12, (2.8)], it is straightforward to show that $\mathcal{P}^{f}(R)$ is an injective cogenerator and a projective generator for $\mathcal{G}^{\prime}(R)$ and that $\mathcal{G}^{\prime}(R)$ is closed under extensions. Theorem 4.9 now implies $\mathcal{G}^{n}\left(\mathcal{G}^{\prime}(R)\right) \subseteq \mathcal{G}(R)$ for each $n \geqslant 1$.

\section{ACKNOWLEDGMENTS}

We are indebted to the referee for his/her careful reading of this work. 


\section{REFERENCES}

1. M. Auslander, Anneaux de Gorenstein, et torsion en algèbre commutative, Séminaire d'Algèbre Commutative dirigé par Pierre Samuel, vol. 1966/67, Secrétariat mathématique, Paris, 1967. MR 37 \#1435

2. M. Auslander and M. Bridger, Stable module theory, Memoirs of the American Mathematical Society, No. 94, American Mathematical Society, Providence, R.I., 1969. MR 42 \#4580

3. M. Auslander and R.-O. Buchweitz, The homological theory of maximal Cohen-Macaulay approximations, Mém. Soc. Math. France (N.S.) (1989), no. 38, 5-37, Colloque en l'honneur de Pierre Samuel (Orsay, 1987). MR 1044344 (91h:13010)

4. L. L. Avramov and H.-B. Foxby, Ring homomorphisms and finite Gorenstein dimension, Proc. London Math. Soc. (3) 75 (1997), no. 2, 241-270. MR 98d:13014

5. R.-O. Buchweitz and H. Flenner, Power series rings and projectivity, Manuscripta Math. 119 (2006), no. 1, 107-114. MR 2194381 (2007a:13025)

6. L. W. Christensen, Gorenstein dimensions, Lecture Notes in Mathematics, vol. 1747, SpringerVerlag, Berlin, 2000. MR 2002e:13032

7. - Semi-dualizing complexes and their Auslander categories, Trans. Amer. Math. Soc. 353 (2001), no. 5, 1839-1883. MR 2002a:13017

8. E. E. Enochs and O. M. G. Jenda, Gorenstein injective and projective modules, Math. Z. 220 (1995), no. 4, 611-633. MR 1363858 (97c:16011)

9. $ـ$ Relative homological algebra, de Gruyter Expositions in Mathematics, vol. 30, Walter de Gruyter \& Co., Berlin, 2000. MR 1753146 (2001h:16013)

10. H.-B. Foxby, Gorenstein modules and related modules, Math. Scand. 31 (1972), 267-284 (1973). MR 48 \#6094

11. A. Frankild and S. Sather-Wagstaff, Detecting completeness from Ext-vanishing, Proc. Amer. Math. Soc., to appear, arXiv:math.AC/0606736.

12. A. A. Gerko, On homological dimensions, Mat. Sb. 192 (2001), no. 8, 79-94, translation in Sb. Math. 192 (2001), no. 7-8, 1165-1179. MR 2002h:13024

13. E. S. Golod, G-dimension and generalized perfect ideals, Trudy Mat. Inst. Steklov. 165 (1984), 62-66, Algebraic geometry and its applications. MR 85m:13011

14. H. Holm, Gorenstein homological dimensions, J. Pure Appl. Algebra 189 (2004), no. 1, 167193. MR 2038564 (2004k:16013)

15. H. Holm and P. Jørgensen, Semi-dualizing modules and related Gorenstein homological dimensions, J. Pure Appl. Algebra 205 (2006), no. 2, 423-445. MR 2203625

16. H. Holm and D. White, Foxby equivalence over associative rings, J. Math. Kyoto Univ., to appear, arXiv:math.AC/0611838.

17. C. U. Jensen, On the vanishing of $\lim ^{(i)}$, J. Algebra 15 (1970), 151-166. MR 0260839 (41 \#5460)

18. R. Takahashi and D. White, Homological aspects of semidualizing modules, preprint (2007), arXiv: math. AC/0703643.

19. W. V. Vasconcelos, Divisor theory in module categories, North-Holland Publishing Co., Amsterdam, 1974, North-Holland Mathematics Studies, No. 14, Notas de Matemática No. 53. [Notes on Mathematics, No. 53]. MR 0498530 (58 \#16637)

20. D. White, Gorenstein projective dimension with respect to a semidualizing module, preprint (2006), arXiv:math.AC/0611711. 
Sean Sather-Wagstaff, Department of Mathematical Sciences, Kent State UniverSity, Mathematics and Computer Science Building, Summit Street, Kent OH 44242, USA

E-mail address: sather@math.kent.edu

URL: http://www.math.kent.edu/ ${ }^{\text {sather }}$

Tirdad Sharif, School of Mathematics, Institute for Studies in Theoretical Physics and Mathematics, P. O. Box 19395-5746, Tehran, Iran

E-mail address: sharif@ipm.ir

URL: http://www.ipm.ac.ir/IPM/people/personalinfo.jsp?PeopleCode=IP0400060

Diana White, Department of Mathematics, University of Nebraska, 203 Avery Hall, LinCOLN, NE, 68588-0130 USA

E-mail address: dwhite@math.unl.edu

URL: http://www.math.unl.edu/ ${ }^{\text {s-dwhite14/ }}$ 